\title{
Human Milk Oligosaccharide Profiles and Associations with Maternal Nutritional Factors: A Scoping Review
}

\author{
Caren Biddulph ${ }^{1, * \mathbb{C}}$, Mark Holmes ${ }^{1}{ }^{\mathbb{D}}$, Anna Kuballa ${ }^{1}$, Peter S. W. Davies ${ }^{2}$, Pieter Koorts ${ }^{3}$, Roger J. Carter ${ }^{4}$ \\ and Judith Maher ${ }^{1} \mathbb{B}$ \\ 1 School of Health and Behavioural Sciences, University of the Sunshine Coast, Maroochydore DC, \\ Queensland 4558, Australia; MHolmes@usc.edu.au (M.H.); akuballa@usc.edu.au (A.K.); \\ jmaher@usc.edu.au (J.M.) \\ 2 Child Health Research Centre, University of Queensland (UQ), St Lucia, Queensland 4072, Australia; \\ ps.davies@uq.edu.au \\ 3 Department of Neonatology, Royal Brisbane and Women's Hospital, Herston, Queensland 4029, Australia; \\ pieter.koorts@health.qld.gov.au \\ 4 Liaison Librarian, Science, Health, Nursing and Midwifery, and Sport Sciences, University of the Sunshine \\ Coast, Maroochydore DC, Queensland 4558, Australia; rcarter@usc.edu.au \\ * Correspondence: carenbiddulph@gmail.com; Tel.: +61-0466-415-553
}

check for updates

Citation: Biddulph, C.; Holmes, M.; Kuballa, A.; Davies, P.S.W.; Koorts, P.; Carter, R.J.; Maher, J. Human Milk Oligosaccharide Profiles and Associations with Maternal Nutritional Factors: A Scoping Review. Nutrients 2021, 13, 965. https://doi.org/10.3390/nu13030965

Academic Editors: Simon J. S. Cameron and Natalie Shenker

Received: 17 February 2021

Accepted: 12 March 2021

Published: 17 March 2021

Publisher's Note: MDPI stays neutral with regard to jurisdictional claims in published maps and institutional affiliations.

Copyright: (c) 2021 by the authors. Licensee MDPI, Basel, Switzerland. This article is an open access article distributed under the terms and conditions of the Creative Commons Attribution (CC BY) license (https:// creativecommons.org/licenses/by/ $4.0 /)$.

\begin{abstract}
Human milk oligosaccharides (HMOs) are complex unconjugated glycans associated with positive infant health outcomes. This study has examined current knowledge of the effect of maternal diet and nutritional status on the composition of HMOs in breast milk. Using the PRISMA-ScR guidelines, a comprehensive, systematic literature search was conducted using Scopus, Web of Science, Global Health (CABI), and MEDLINE. Titles and abstracts were screened independently by two reviewers against predefined inclusion and exclusion criteria. Fourteen studies met the inclusion criteria and reported on maternal dietary intake $(n=3)$, maternal body composition indices $(n=9)$, and dietary supplementation interventions $(n=2)$. In total, data from 1388 lactating mothers (4011 milk samples) were included. Design methodologies varied substantially across studies, particularly for milk sample collection, HMO analysis, dietary and body composition assessment. Overall, this review has identified potential associations between maternal dietary intake and nutritional status and the HMO composition of human milk, though an abundance and sufficiency of evidence is lacking. Standardised procedures for human milk sample collection and HMO analysis, along with robust and validated nutrition assessment techniques, should be employed to further investigate the impact of maternal nutritional factors on HMO composition.
\end{abstract}

Keywords: maternal diet; maternal body composition; human milk oligosaccharide; breastfeeding

\section{Introduction}

Breast milk is a unique and ideal source of nutrition for most infants [1]. It is comprised of essential nutrients in the right proportions and contains beneficial bioactive factors such as antibodies, hormones and over 200 defined human milk oligosaccharides (HMOs) [1]. HMOs are structurally diverse and complex unconjugated glycans and represent the third largest solid component of human milk after lactose and lipids [2,3]. Their presence in human milk is associated with many positive infant health outcomes, and they play an important role in influencing the development of the infant immune system and gut microbiome [2,4]. In the infant intestinal tract, HMOs act as prebiotics and have selective anti-adhesive, antimicrobial properties [2,5]. As a potential dietary source of sialic acid, some of the sialylated HMOs may be important for infant learning and memory development, though this observation is based primarily on animal studies $[6,7]$.

Whilst there has been significant research into the physiological and immunological properties of certain HMOs, little is currently understood about the maternal factors that 
affect the composition of oligosaccharides in human milk, particularly those not associated with genetic influences. HMO concentrations and composition in the milk of lactating women shows substantial intra- and inter-individual differences [8]. The total $\mathrm{HMO}$ content in milk decreases over the course of lactation, with colostrum containing 20-25 g/L and mature milk containing approximately 5-15 g/L [9]. Differential compositional changes also take place over time; levels of fucosylated HMOs are highest early in lactation and most other HMOs appear to decrease, or remain quite constant $[8,10]$. The variation in HMO composition is primarily due to maternal genetic factors, with numerous studies reporting on the effects of polymorphism of the Secretor and Lewis genes on the amount and diversity of HMOs $[2,9,11]$. Lactating women are often classified as Secretors or non-secretors based upon the presence or absence of the fucosyltransferase 2 ('FUT2') gene, with Secretor mothers producing more neutral $\alpha 1-2$-fucosylated HMOs in their milk [9]. Emerging research indicates that other interrelated and somewhat complex factors may influence the composition of HMOs [12], including parity and mode of delivery, environment influences such as geographic location and seasonality, and maternal influences such as age and nutritional status [12-22]. There is limited information and no synthesis on the effect of maternal nutritional intake, diet quality, and body composition on HMO composition during lactation [23].

Given the numerous and varied beneficial effects of HMOs on infant health and development $[24,25]$, a better understanding of the influence of maternal dietary factors on $\mathrm{HMO}$ composition is desirable. The aim of this scoping review is to investigate any known associations between HMO composition and maternal nutrition and nutritionrelated factors during lactation. The primary objective of this review is to assess the impact of maternal nutritional intake and patterns, nutritional status, and nutrition-related aspects, such as the maternal microbiome, on the composition of HMOs in breast milk. A brief discussion on hypotheses around possible mechanisms by which these factors may influence HMO profiles will be included. This review is intended for general clinicians, nutritionists, dietitians, neonatologists, metabolomics researchers, analytical chemists, and those working in lactation research and donor milk banks.

\section{Materials and Methods}

\subsection{Study Design}

This scoping review was planned and conducted using the Preferred Reporting Items for Systematic Reviews and Meta-Analyses guidelines extension for scoping reviews (PRIMSA-ScR) [26].

The review protocol was drafted using the "Preferred Reporting Items for Systematic Reviews and Meta-analysis Protocols" (PRISMA-P) and has been made publicly available; reference: Biddulph, C., Davies, P., Carter, R.J. and Maher, J. (2020). Human milk oligosaccharides profiles and associations with maternal nutritional factors: A scoping review protocol. USC Research Bank. https: / / research.usc.edu.au/discovery / delivery?vid=61 USC_INST:ResearchRepository\&repId=12126689620002621\#13129825250002621 (accessed on 15 March 2021).

\subsection{Identifying the Research Question}

We aimed to address the following question: "What is known about the influence of maternal dietary intake, nutritional supplementation and body composition on the composition of HMOs in breastmilk?".

\subsection{Search Strategy and Eligibility Criteria}

Two authors (C.B. and J.M.) performed concurrent and comprehensive systematic electronic literature searches to find relevant studies reporting on the links between maternal nutritional factors and HMOs. Recommended health and nutrition electronic databases were searched on 17 February 2020 at 13:00 AEST. These databases included: Scopus, Web 
of Science, Global Health (CABI) and MEDLINE (incl. PubMed). The following search strategy was applied to terms listed within the titles, abstracts, and keywords of articles:

(maternal OR mother OR human OR female) AND (diet* OR supplement* OR nutri* OR macronutrient* OR carbohydrate OR sugar* OR protein OR fat* OR fibre OR fiber OR prebiotic* OR probiotic* OR "body composition" OR bmi OR weight OR microbiome* OR bacteria* OR "entero-mammary pathway" OR lact*) AND (HMO OR "human milk oligosaccharide" $\mathrm{OR}$ "breastmilk oligosaccharide").

A research librarian (R.C.) assisted in advising on the search terms and eligibility criteria. The review includes original research papers that investigated associations between HMO content or profiles and at least one maternal factor that is nutrition-related, such as dietary intake, supplementation, nutritional status, and body composition. We selected studies in humans published as full-length articles and excluded conference abstracts, editorials, letters to the editor and case reports. We included randomised controlled trials, observational cohort studies, cross-sectional observational studies, and excluded reviews of mixed methods studies. Outcomes that were assessed included the total HMO content and absolute concentrations of single HMOs. There was no time exclusion on publication dates.

The final search results were exported into Endnote ${ }^{\circledR}$ reference management software, and duplicates were removed. The process was repeated for subsequent databases and sources, with articles sorted into folders and details captured as per the PRISMA flow chart (Figure 1). These results were supplemented by articles found using methods such as citation searching of relevant articles, snowballing, and reference list searching.

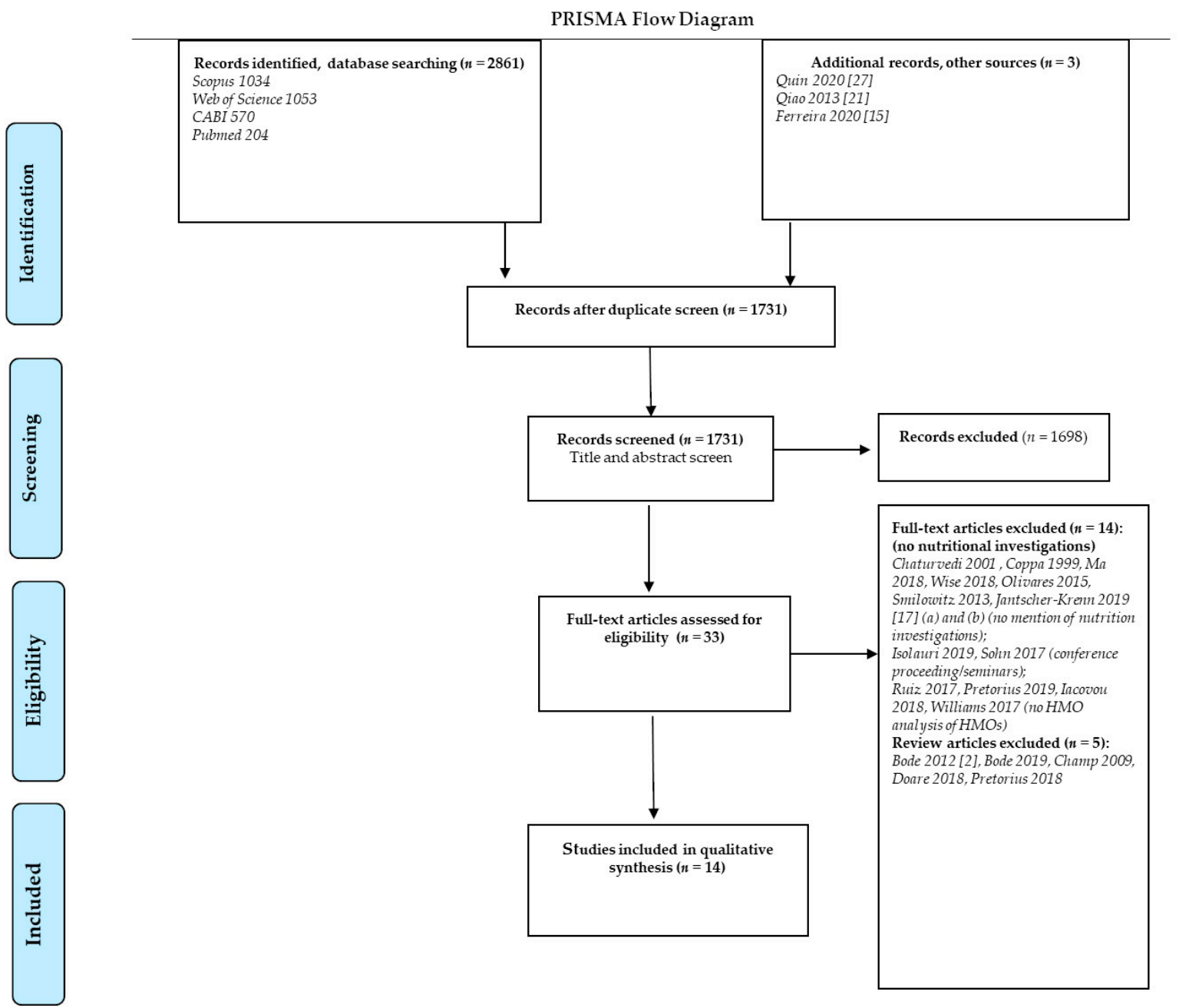

Figure 1. Flow diagram describing study selection process $[2,15,17,21,27]$. 


\subsection{Article Screening and Data Abstraction}

Two authors (C.B. and J.M.) screened the titles and abstracts of all papers and performed article selection independently according to the inclusion and exclusion criteria.

Data were extracted from the full-text papers by C.B. using a predefined data collection proforma, and subsequently reviewed by J.M. Information was collected on study characteristics and design, location, description of participants and sample size, milk sample collection, maternal nutrition assessment measures, $\mathrm{HMO}$ analysis, and main outcomes and conclusions. Data abstraction details are included in Table 1.

Table 1. Studies evaluating the possible effects of maternal nutrition-related factors on HMO content and profiles.

\begin{tabular}{|c|c|c|c|c|c|c|c|}
\hline Reference & $\begin{array}{c}\text { Exposure } \\
\text { Variables of } \\
\text { Interest }\end{array}$ & Study Design & $\begin{array}{c}\text { Population } \\
\text { and Sample } \\
\text { Number }\end{array}$ & $\begin{array}{c}\text { Maternal } \\
\text { Nutritional } \\
\text { Assessment } \\
\text { Tool }\end{array}$ & $\begin{array}{c}\text { Milk } \\
\text { Sampling }\end{array}$ & $\begin{array}{c}\text { HMO } \\
\text { Analysis }\end{array}$ & $\begin{array}{l}\text { Relevant } \\
\text { Main } \\
\text { Outcomes }\end{array}$ \\
\hline Azad et al. [12] & $\begin{array}{c}\text { Maternal } \\
\text { pre-pregnancy } \\
\text { BMI and usual } \\
\text { dietary intake } \\
\text { during } \\
\text { pregnancy. }\end{array}$ & $\begin{array}{c}\text { Prospective } \\
\text { observational } \\
\text { study. }\end{array}$ & $\begin{array}{c}n=427 \text { milk } \\
\text { samples, } 1 \\
\text { from each } \\
\text { mother-infant } \\
\text { dyad (healthy } \\
\text { term infants, } \\
\text { Canadian } \\
\text { multi-ethnic } \\
\text { mothers). }\end{array}$ & $\begin{array}{c}\text { Validated FFQ } \\
\text { during } \\
\text { pregnancy; } \\
\text { pre-pregnancy } \\
\text { BMI } \\
\text { (self-reported } \\
\text { weight, } \\
\text { measured } \\
\text { height). }\end{array}$ & $\begin{array}{l}\text { Combined and } \\
\text { refrigerated } \\
\text { single sample } \\
\text { from multiple } \\
\text { feeds during a } \\
\text { 24-h period at } \\
\text { 3-4 months } \\
\text { postpartum } \\
\text { (median: } 16 \\
\text { weeks, IQR: } \\
\text { 14-19 weeks). }\end{array}$ & $\begin{array}{l}\text { HPLC; } 19 \\
\text { HMOs; total } \\
\text { and relative } \\
\text { abundance of } \\
\text { HMOs; total } \\
\text { HMO-bound } \\
\text { fucose and } \\
\text { sialic acid; } \\
\text { FUT2 “Secretor } \\
\text { status" defined } \\
\text { by 2'-FL. }\end{array}$ & $\begin{array}{c}\text { Maternal } \\
\text { dietary intake } \\
\text { and BMI not } \\
\text { correlated with } \\
\text { HMO concen- } \\
\text { trations. }\end{array}$ \\
\hline $\begin{array}{c}\text { Davis et al. } \\
{[14]}\end{array}$ & $\begin{array}{c}\text { Seasonal } \\
\text { environmental } \\
\text { changes as } \\
\text { proxy for } \\
\text { caloric intake. }\end{array}$ & $\begin{array}{l}\text { Prospective } \\
\text { observational } \\
\text { study. }\end{array}$ & $\begin{array}{c}n=99 \text { milk } \\
\text { samples, from } \\
33 \\
\text { mother-infant } \\
\text { dyads (rural } \\
\text { African } \\
\text { Gambian } \\
\text { women). }\end{array}$ & $\begin{array}{l}\text { Maternal body } \\
\text { weight. Sea- } \\
\text { son/environment } \\
\text { as proxy for } \\
\text { caloric intake. }\end{array}$ & $\begin{array}{c}33 \text { samples } \times 3 \\
\text { time points } \\
\text { (postpartum } \\
\text { weeks } 4,16, \\
\text { 20); } 5 \mathrm{~mL} \text { of } \\
\text { hand- } \\
\text { expressed milk } \\
\text { from each } \\
\text { breast into a } \\
\text { separate tube } \\
\text { in the morning. } \\
\text { Start, middle } \\
\text { or end of feed } \\
\text { not specified. }\end{array}$ & $\begin{array}{c}\text { Nano-HPLC- } \\
\text { Chip/TOF } \\
\text { Mass } \\
\text { Spectrometry; } \\
19 \text { HMOs. } \\
\text { Total HMOs, } \\
\text { Fucosylated, } \\
\text { sialylated, } \\
\text { undecorated } \\
\text { HMOs. } \\
\text { Mothers with } \\
\text { less than } \\
\text { Secretors }>6 \% \\
\text { relative } \alpha(1-2) \\
\text { fucosylation. }\end{array}$ & $\begin{array}{l}\text { HMO levels } \\
\text { are associated } \\
\text { with season. } \\
\text { HMO concen- } \\
\text { trations / total } \\
\text { amount } \\
\text { significantly } \\
\text { higher ( } p= \\
\text { 0.01) in dry } \\
\text { season (food is } \\
\text { more plentiful } \\
\text { and hence total } \\
\text { dietary energy } \\
\text { intake may be } \\
\text { greater). }\end{array}$ \\
\hline $\begin{array}{c}\text { Ferreira et al. } \\
{[15]}\end{array}$ & $\begin{array}{l}\text { Maternal an- } \\
\text { thropometric } \\
\text { characteristics. }\end{array}$ & $\begin{array}{c}\text { Prospective } \\
\text { longitudinal } \\
\text { observational } \\
\text { study. }\end{array}$ & $\begin{array}{c}n=174 \text { milk } \\
\text { samples from } \\
101 \text { subjects } \\
\text { (healthy, Latin- } \\
\text { American, } \\
\text { pregnant } \\
\text { women). }\end{array}$ & $\begin{array}{c}\text { Pre-pregnancy } \\
\text { BMI } \\
\text { (self-reported } \\
\text { pre-pregnancy } \\
\text { weight and } \\
\text { measured } \\
\text { height), } \\
\text { gestational } \\
\text { weight gain } \\
\text { (weighed at } \\
\text { last prenatal } \\
\text { visit); } \\
\text { Supplement } \\
\text { use via } \\
\text { questionnaire } \\
\text { (iron and folic } \\
\text { acid). }\end{array}$ & $\begin{array}{c}\text { Manual } \\
\text { expression of } \\
\text { milk samples } \\
\text { at } 2-8 \text { days, } \\
28-50 \text { and } \\
\text { 88-119 days, in } \\
\text { the morning } \\
\text { after breakfast. }\end{array}$ & $\begin{array}{l}\text { HPLC with } \\
\text { fluorescence } \\
\text { detection } \\
\text { (HPLC-FL); } 19 \\
\text { HMOs. HMO } \\
\text { absolute (total) } \\
\text { and relative } \\
\text { abundances. } \\
\text { Secretor status } \\
\text { determined by } \\
\text { presence of } \\
\text { 2'-FL and } \\
\text { LNFP I. }\end{array}$ & $\begin{array}{l}\text { Maternal } \\
\text { pre-pregnancy } \\
\text { weight and } \\
\text { BMI is } \\
\text { associated with } \\
\text { HMO } \\
\text { composition } \\
\text { (low-moderate } \\
\text { Spearman } \\
\text { correlation } \\
\text { values; } \\
\text { positively } \\
\text { correlated with } \\
\text { LNnT (0.4) and } \\
\text { inversely } \\
\text { correlated with } \\
\text { LNFP III } \\
(-0.4) \text { ). }\end{array}$ \\
\hline
\end{tabular}


Table 1. Cont.

\begin{tabular}{|c|c|c|c|c|c|c|c|}
\hline Reference & $\begin{array}{c}\text { Exposure } \\
\text { Variables of } \\
\text { Interest }\end{array}$ & Study Design & $\begin{array}{c}\text { Population } \\
\text { and Sample } \\
\text { Number }\end{array}$ & $\begin{array}{c}\text { Maternal } \\
\text { Nutritional } \\
\text { Assessment } \\
\text { Tool }\end{array}$ & $\begin{array}{c}\text { Milk } \\
\text { Sampling }\end{array}$ & $\begin{array}{c}\text { HMO } \\
\text { Analysis }\end{array}$ & $\begin{array}{c}\text { Relevant } \\
\text { Main } \\
\text { Outcomes }\end{array}$ \\
\hline $\begin{array}{c}\text { Gridneva et al. } \\
\text { [16] }\end{array}$ & $\begin{array}{c}\text { Maternal body } \\
\text { composition } \\
\text { indices } \\
\text { (fat-free and fat } \\
\text { mass, } \\
\text { percentage fat } \\
\text { mass (\%FM). }\end{array}$ & $\begin{array}{l}\text { Longitudinal } \\
\text { proof of } \\
\text { concept/pilot } \\
\text { study. }\end{array}$ & $\begin{array}{c}n=80 \text { milk } \\
\text { samples, from } \\
20 \\
\text { mother-infant } \\
\text { dyads (healthy } \\
\text { term infants } \\
\text { and Australian, } \\
\text { predominantly } \\
\text { Caucasian } \\
\text { mothers). }\end{array}$ & $\begin{array}{c}\text { Maternal body } \\
\text { weight, BMI, } \\
\text { and body } \\
\text { composition } \\
\text { indices at } 4 \\
\text { visits utilising } \\
\text { BIS. }\end{array}$ & $\begin{array}{l}\text { Small }(1-2 \mathrm{~mL}) \\
\text { pre-/post feed } \\
\text { milk samples } \\
\text { were collected } \\
\text { into } 5 \mathrm{~mL} \\
\text { polypropylene } \\
\text { vials when } \\
\text { infants were } 2 \\
\text { and/or } 5,9, \\
\text { and } 12 \\
\text { months-old. }\end{array}$ & $\begin{array}{c}\text { None (Total } \\
\text { HMO } \\
\text { concentration } \\
\text { (g/L) } \\
\text { estimated by } \\
\text { deducting } \\
\text { lactose } \\
\text { concentration } \\
\text { from total } \\
\text { carbohydrate } \\
\text { concentration). } \\
\text { No } \\
\text { determination } \\
\text { of Secretor } \\
\text { status. }\end{array}$ & $\begin{array}{c}\text { No } \\
\text { associations } \\
\text { with maternal } \\
\text { body } \\
\text { composition; } \\
\text { prior to FDR } \\
\text { adjustment, } \\
\text { higher HMO } \\
\text { calculated } \\
\text { daily intake } \\
\text { was associated } \\
\text { with higher } \\
\text { maternal \%FM } \\
\text { and FM/FFM } \\
\text { between } 2 \text { and } \\
5 \text { months, and } \\
\text { with lower } \\
\text { maternal \%FM } \\
\text { and FM/FFM } \\
\text { at } 9 \text { and } 12 \\
\text { months. }\end{array}$ \\
\hline $\begin{array}{c}\text { Isganaitiset al. } \\
{[28]}\end{array}$ & $\begin{array}{c}\text { Maternal } \\
\text { obesity and } \\
\text { postnatal } \\
\text { weight gain. }\end{array}$ & $\begin{array}{l}\text { Prospective } \\
\text { observational } \\
\text { study. }\end{array}$ & $\begin{array}{c}n=57 \text { total } \\
\text { milk samples; } \\
\text { from } 31 \\
\text { mothers at } 1 \\
\text { month and } 26 \\
\text { at } 6 \text { months } \\
\text { (American, } \\
\text { predominantly } \\
\text { Caucasian } \\
\text { women). }\end{array}$ & $\begin{array}{c}\text { Maternal } \\
\text { weight, height, } \\
\text { BMI at study } \\
\text { site by } \\
\text { researchers. } \\
\text { Participants } \\
\text { grouped by } \\
\text { maternal } \\
\text { pre-pregnancy } \\
\text { BMI ( } n=15 \\
\text { BMI }<25, \text { lean, } \\
n=16 \text { BMI } \geq \\
25 \text { kg/m², } \\
\text { over- } \\
\text { weight/obese). } \\
\text { Gestational } \\
\text { weight gain, } \\
\text { BMI at } 1 \text { month } \\
\text { postpartum. }\end{array}$ & $\begin{array}{c}\text { Complete } \\
\text { expression of a } \\
\text { single breast } \\
\text { (right } \\
\text { preferred) at } 1 \\
\text { and } 6 \text { months } \\
\text { postpartum } \\
\text { using an } \\
\text { electric pump. } \\
\text { 2-2.5 h after } \\
\text { last feed } \\
\text { between } 8 \text { and } \\
10 \text { am (around } \\
\text { midday). }\end{array}$ & $\begin{array}{c}\text { Untargeted } \\
\text { metabolomics } \\
\text { analysis using } \\
\text { LC-GCMS. } \\
\text { Reported on } 3 \\
\text { oligosaccha- } \\
\text { rides: } \\
\text { 2'-FL, LNFP I, } \\
\text { and LNFP } \\
\text { II/III. No } \\
\text { determination } \\
\text { of Secretor } \\
\text { status. }\end{array}$ & $\begin{array}{c}\text { Maternal } \\
\text { obesity was } \\
\text { linked to } \\
\text { differences in } \\
\text { HMO } \\
\text { composition at } \\
1 \text { month } \\
\text { postpartum } \\
\text { (2'-FL, LNFP I } \\
\text { and LNFP } \\
\text { II/III } \\
\text { significantly } \\
\text { correlated with } \\
\text { maternal BMI). }\end{array}$ \\
\hline $\begin{array}{c}\text { Jorgensenet al. } \\
{[18]}\end{array}$ & $\begin{array}{l}\text { Lipid-based } \\
\text { Nutrient (LNS) } \\
\text { or multiple } \\
\text { micronutrient } \\
\text { (MMN) } \\
\text { supplements, } \\
\text { compared with } \\
\text { iron and folic } \\
\text { acid during } \\
\text { pregnancy and } \\
\text { placebo } \\
\text { postpartum. } \\
\text { Potential } \\
\text { covariates } \\
\text { included } \\
\text { baseline } \\
\text { maternal BMI } \\
\text { (in kg/m2). }\end{array}$ & $\begin{array}{c}\text { Randomised, } \\
\text { single } \\
\text { (assessor)- } \\
\text { blind, parallel } \\
\text { group- } \\
\text { controlled } \\
\text { supplementa- } \\
\text { tion trial. } \\
\text { Outcomes } \\
\text { assessed } \\
\text { according to } \\
\text { intention to } \\
\text { treat principle. }\end{array}$ & $\begin{array}{c}n=645 \\
\text { breastmilk } \\
\text { samples (rural } \\
\text { Malawian } \\
\text { (African) } \\
\text { women, low } \\
\text { socio- } \\
\text { economic } \\
\text { status). }\end{array}$ & $\begin{array}{l}\text { Adherence } \\
\text { index for } \\
\text { supplement } \\
\text { compliance. } \\
\text { Weight and } \\
\text { height in } \\
\text { triplicate by } \\
\text { trained anthro- } \\
\text { pometrists at } \\
\text { enrollment to } \\
\text { calculate BMI } \\
\text { during } \\
\text { pregnancy } \\
\text { ( } 20 \text {, at } 32 \text { and } \\
36 \text { gestational } \\
\text { weeks) and } \\
\text { once after birth } \\
\text { (at } 1-2 \text { weeks } \\
\text { after delivery). }\end{array}$ & $\begin{array}{c}\text { Single sample, } \\
\text { manual } \\
\text { expression of } \\
\text { the full content } \\
\text { of one breast } \\
\text { into a sterile } \\
\text { plastic cup at } 6 \\
\text { months } \\
\text { postpartum. }\end{array}$ & $\begin{array}{c}\text { Nano-LC } \\
\text { microfluidic } \\
\text { chip coupled } \\
\text { to electrospray } \\
\text { time-of flight } \\
\text { mass } \\
\text { spectrometer. } \\
\text { Summed total } \\
\text { HMOs, } \\
\text { fucosylated, } \\
\text { sialylated and } \\
\text { nonfucosy- } \\
\text { lated neutral } \\
\text { glycans. No } \\
\text { determination } \\
\text { of Secretor } \\
\text { status. }\end{array}$ & $\begin{array}{l}\text { Supplementation } \\
\text { with an LNS or } \\
\text { MMN capsule } \\
\text { during } \\
\text { pregnancy and } \\
\text { postpartum } \\
\text { did not } \\
\text { increase HMO } \\
\text { or bioactive } \\
\text { milk proteins. } \\
\text { No interactions } \\
\text { or group } \\
\text { differences in } \\
\text { HMOs } \\
\text { according to } \\
\text { maternal BMI. }\end{array}$ \\
\hline
\end{tabular}


Table 1. Cont.

\begin{tabular}{|c|c|c|c|c|c|c|c|}
\hline Reference & $\begin{array}{l}\text { Exposure } \\
\text { Variables of } \\
\text { Interest }\end{array}$ & Study Design & $\begin{array}{l}\text { Population } \\
\text { and Sample } \\
\text { Number }\end{array}$ & $\begin{array}{c}\text { Maternal } \\
\text { Nutritional } \\
\text { Assessment } \\
\text { Tool }\end{array}$ & $\begin{array}{c}\text { Milk } \\
\text { Sampling }\end{array}$ & $\begin{array}{c}\text { HMO } \\
\text { Analysis }\end{array}$ & $\begin{array}{l}\text { Relevant } \\
\text { Main } \\
\text { Outcomes }\end{array}$ \\
\hline $\begin{array}{c}\text { Larsson, et al. } \\
\text { [19] }\end{array}$ & $\begin{array}{c}\text { Maternal } \\
\text { pre-pregnancy } \\
\text { BMI, } \\
\text { gestational } \\
\text { weight gain, } \\
\text { maternal } \\
\text { weight at } 5 \text { and } \\
9 \text { months } \\
\text { postpartum. }\end{array}$ & $\begin{array}{l}\text { Prospective } \\
\text { observational } \\
\text { cohort study. }\end{array}$ & $\begin{array}{c}n=60 \text { milk } \\
\text { samples; from } \\
30 \\
\text { mother-infant } \\
\text { dyads (13 } \\
\text { high-weight } \\
\text { infants and } 17 \\
\text { normal-weight } \\
\text { healthy Danish } \\
\text { infants). }\end{array}$ & $\begin{array}{l}\text { Maternal } \\
\text { pre-pregnancy } \\
\text { BMI and } \\
\text { gestational } \\
\text { weight gain } \\
\text { self-reported; } \\
\text { Maternal } \\
\text { weight and } \\
\text { height } \\
\text { measured } \\
\text { using } \\
\text { standardized } \\
\text { procedure at } \\
\text { the infant's age } \\
\text { 5- and } \\
\text { 9-months } \\
\text { visits. }\end{array}$ & $\begin{array}{l}\text { Well-mixed } \\
\text { samples of } \\
\text { right and left } \\
\text { breasts: } \\
\text { mothers were } \\
\text { asked to pump } \\
\text { the entire } \\
\text { content of both } \\
\text { breasts using a } \\
\text { manual breast } \\
\text { pump at } 5 \text { and } \\
9 \text { months } \\
\text { postpartum. }\end{array}$ & $\begin{array}{l}\text { HPLC after } \\
\text { fluorescent } \\
\text { derivatization; } \\
19 \text { HMOs, total } \\
\text { HMO-bound } \\
\text { fucose and } \\
\text { sialic acid, total } \\
\text { HMO. } \\
\text { Secretor status } \\
\text { was } \\
\text { determined } \\
\text { based on } \\
\text { presence or } \\
\text { near-absence } \\
\text { of } 2^{\prime} \text {-FL and } \\
\text { LNFP I. }\end{array}$ & $\begin{array}{c}\text { Gestational } \\
\text { weight gain } \\
\text { was not } \\
\text { associated with } \\
\text { HMO. } \\
\text { Maternal BMI } \\
\text { at } 5 \text { months } \\
\text { postpartum } \\
\text { was positively } \\
\text { with 2'-FL, } \\
\text { total HMO and } \\
\text { total } \\
\text { HMO-bound } \\
\text { fucose; and } \\
\text { negatively } \\
\text { associated with } \\
6^{\prime} \text {-SL and LSTb } \\
\text { (all } p \leq 0.03 \text { ). } \\
\text { Weak } \\
\text { associations } \\
\text { between HMO } \\
\text { and Maternal } \\
\text { pre-pregnancy } \\
\text { BMI. }\end{array}$ \\
\hline $\begin{array}{l}\text { McGuire et al. } \\
\text { [20] }\end{array}$ & $\begin{array}{l}\text { Maternal an- } \\
\text { thropometric } \\
\text { indices } \\
\text { (weight, height, } \\
\text { BMI). }\end{array}$ & $\begin{array}{c}\text { Cross- } \\
\text { sectional, } \\
\text { epidemiologic } \\
\text { cohort study } \\
\text { that involved } \\
\text { multiple (11) } \\
\text { international } \\
\text { sites. }\end{array}$ & $\begin{array}{c}n=410 \text { milk } \\
\text { samples; } 1 \\
\text { from each } \\
\text { healthy, } \\
\text { breastfeeding } \\
\text { woman; } \\
\text { multisite: } 40, \\
40,40,40,40, \\
\text { 42, 43, 41, 24, } \\
\text { 41, and 19 } \\
\text { women from } \\
\text { rural Ethiopia, } \\
\text { urban Ethiopia, } \\
\text { rural Gambia, } \\
\text { urban Gambia, } \\
\text { Ghana, Kenya, } \\
\text { Peru, Spain, } \\
\text { Sweden, USA } \\
\text { Washington, } \\
\text { and USA } \\
\text { California. }\end{array}$ & $\begin{array}{c}\text { Maternal body } \\
\text { weight, height } \\
\text { via } \\
\text { questionnaire } \\
\text { (self-reported); } \\
\text { BMI calculated } \\
\text { upon } \\
\text { enrolment (2 } \\
\text { weeks -5 } \\
\text { months } \\
\text { postpar- } \\
\text { tum/during } \\
\text { lactation). }\end{array}$ & $\begin{array}{c}1 \text { breast only; } \\
\leq 200 \mathrm{~mL} \\
\text { (typically } \\
40-60 \mathrm{~mL} \text { ), } \\
\text { manually } \\
\text { expressed or } \\
\text { with a breast } \\
\text { pump; at } 2 \\
\text { weeks-5 } \\
\text { months } \\
\text { postpartum. }\end{array}$ & $\begin{array}{l}\text { HPLC-MS; } 19 \\
\text { HMOs. } \\
\text { Proportion of } \\
\text { each HMO and } \\
\text { the total } \\
\text { concentration } \\
\text { of HMOs as } \\
\text { the sum of the } \\
\text { annotated } \\
\text { oligosaccha- } \\
\text { rides. } \\
\text { Secretor milk } \\
\text { was defined as } \\
\text { having a 2'-FL } \\
\text { concentration } \\
\text { that was } \\
\text { greater than a } \\
\text { natural, very } \\
\text { low break in } \\
\text { the data. }\end{array}$ & $\begin{array}{c}\text { Maternal } \\
\text { weight and } \\
\text { BMI were } \\
\text { positively } \\
\text { correlated with } \\
2^{\prime} \text {-FL }(\mathrm{r}=0.20) \text {, } \\
\text { FLNH }(\mathrm{r}=0.19 \\
\text { and } 0.15, \\
\text { respectively). } \\
\text { Maternal } \\
\text { weight was } \\
\text { positively } \\
\text { correlated with } \\
\text { LNFP III } \\
\text { (r }=0.20) \text { and } \\
\text { DFLNT } \\
\text { ( } \mathrm{r}=0.14) . \\
\text { Maternal } \\
\text { weight and } \\
\text { BMI were } \\
\text { inversely } \\
\text { correlated with } \\
\text { LNnT and } \\
\text { DSLNT } \\
\text { (r = 20.16 and } \\
20.21, \\
\text { respectively; } \\
\text { and } \mathrm{r}=20.20 \\
\text { and } 20.24, \\
\text { respectively). }\end{array}$ \\
\hline
\end{tabular}


Table 1. Cont.

\begin{tabular}{|c|c|c|c|c|c|c|c|}
\hline Reference & $\begin{array}{c}\text { Exposure } \\
\text { Variables of } \\
\text { Interest }\end{array}$ & Study Design & $\begin{array}{c}\text { Population } \\
\text { and Sample } \\
\text { Number }\end{array}$ & $\begin{array}{c}\text { Maternal } \\
\text { Nutritional } \\
\text { Assessment } \\
\text { Tool }\end{array}$ & $\begin{array}{c}\text { Milk } \\
\text { Sampling }\end{array}$ & $\begin{array}{c}\text { HMO } \\
\text { Analysis }\end{array}$ & $\begin{array}{c}\text { Relevant } \\
\text { Main } \\
\text { Outcomes }\end{array}$ \\
\hline $\begin{array}{c}\text { Moossavi et al. } \\
\text { [29] }\end{array}$ & $\begin{array}{l}\text { Maternal body } \\
\text { composition } \\
\text { (BMI) and fish } \\
\text { oil supplement } \\
\text { use during } \\
\text { pregnancy. }\end{array}$ & $\begin{array}{l}\text { Cross sectional } \\
\text { observational } \\
\text { study. (Repre- } \\
\text { sentative } \\
\text { cohort from the } \\
\text { Longitudinal, } \\
\text { population- } \\
\text { based birth } \\
\text { cohort study } \\
\text { (CHILD)). }\end{array}$ & $\begin{array}{c}n=393,1 \\
\text { breastmilk } \\
\text { sample from } \\
\text { representative } \\
\text { subset of } \\
\text { mothers in the } \\
\text { CHILD study } \\
\text { (healthy term } \\
\text { infants, } \\
\text { Canadian } \\
\text { multi-ethnic } \\
\text { mothers). }\end{array}$ & $\begin{array}{l}\text { BMI calculated } \\
\text { (self-reported } \\
\text { weight, } \\
\text { measured } \\
\text { height), Fish } \\
\text { oil supplement } \\
\text { self-reported } \\
\text { by } \\
\text { standardized } \\
\text { questionnaire. }\end{array}$ & $\begin{array}{c}1 \text { sample at 3-4 } \\
\text { months } \\
\text { postpartum } \\
\text { (mean (SD) } 17 \\
\text { (5) weeks } \\
\text { postpartum), } \\
\text { mix of foremilk } \\
\text { and hindmilk } \\
\text { from multiple } \\
\text { feeds during a } \\
\text { 24-h period; } \\
\text { manual/hand } \\
\text { or pump } \\
\text { expression. }\end{array}$ & $\begin{array}{l}\text { HPLC-MS; } 19 \\
\text { HMOs, } \\
\text { summed to } \\
\text { estimate total } \\
\text { HMO } \\
\text { concentration, } \\
\text { HMO-bound } \\
\text { fucose (Fuc) } \\
\text { and } \\
\text { HMO-bound } \\
\text { sialic acid (Sia). } \\
\text { Maternal } \\
\text { secretor status } \\
\text { by the presence } \\
\text { of 2'-FL or } \\
\text { LNFP I. }\end{array}$ & $\begin{array}{l}\text { Maternal diet } \\
\text { and BMI are } \\
\text { interrelated, } \\
\text { and both can } \\
\text { modify gut } \\
\text { microbiota } \\
\text { composition as } \\
\text { well as the } \\
\text { macro- and } \\
\text { micro-nutrient } \\
\text { profile and } \\
\text { microbiota of } \\
\text { human milk } \\
\text { (although } \\
\text { effect sizes } \\
\text { were small } \\
\text { (<2\% of } \\
\text { variation } \\
\text { explained). }\end{array}$ \\
\hline Qiao et al. [21] & $\begin{array}{c}\text { Maternal } \\
\text { dietary intake } \\
\text { during } \\
\text { lactation. }\end{array}$ & $\begin{array}{c}\text { Cross-sectional } \\
\text { observational } \\
\text { study. }\end{array}$ & $\begin{array}{c}n=90,1 \\
\text { breastmilk } \\
\text { sample per } \\
\text { woman } \\
\text { (healthy } \\
\text { Chinese } \\
\text { women with } \\
\text { term infants } \\
(37-42 \text { weeks)). }\end{array}$ & $\begin{array}{l}\text { Validated 72-h } \\
\text { food frequency } \\
\text { questionnaire, } \\
\text { weighed where } \\
\text { possible; } \\
\text { Chinese } \\
\text { Dietary } \\
\text { Reference } \\
\text { Intakes. }\end{array}$ & $\begin{array}{l}1 \text { sample taken } \\
\text { at day } 40( \pm 7) \\
\text { postpartum at } \\
\text { the end of a } \\
\text { breastfeed; } 10 \\
\text { mL within } 15 \\
\text { mins, stored at } \\
-25^{\circ} \mathrm{C} \text {. }\end{array}$ & $\begin{array}{l}\text { HPLC-FLD; } \\
\text { human breast } \\
\text { milk sialic acid } \\
\text { concentrations } \\
\text { (free sialic acid, } \\
\text { bound to } \\
\text { oligosaccha- } \\
\text { rides and } \\
\text { bound to } \\
\text { protein). No } \\
\text { individual } \\
\text { HMO } \\
\text { quantification. }\end{array}$ & $\begin{array}{l}82.35 \% \text { of the } \\
\text { sialic acid in } \\
\text { breastmilk was } \\
\text { found bound } \\
\text { to free oligosac- } \\
\text { charides. } \\
\text { Higher dietary } \\
\text { intake of } \\
\text { Vitamin A (and } \\
\text { of milk, beef, } \\
\text { egg, mutton, } \\
\text { and pork) was } \\
\text { associated with } \\
\text { higher milk } \\
\text { sialic acid } \\
\text { levels } \\
\text { (standardized } \\
\text { coefficients = } \\
0.713 ; p= \\
0.000 \text { ). }\end{array}$ \\
\hline
\end{tabular}


Table 1. Cont.

\begin{tabular}{|c|c|c|c|c|c|c|c|}
\hline Reference & $\begin{array}{c}\text { Exposure } \\
\text { Variables of } \\
\text { Interest }\end{array}$ & Study Design & $\begin{array}{c}\text { Population } \\
\text { and Sample } \\
\text { Number }\end{array}$ & $\begin{array}{c}\text { Maternal } \\
\text { Nutritional } \\
\text { Assessment } \\
\text { Tool }\end{array}$ & $\begin{array}{c}\text { Milk } \\
\text { Sampling }\end{array}$ & $\begin{array}{c}\text { HMO } \\
\text { Analysis }\end{array}$ & $\begin{array}{c}\text { Relevant } \\
\text { Main } \\
\text { Outcomes }\end{array}$ \\
\hline Quin et al. [27] & $\begin{array}{c}\text { Maternal } \\
\text { dietary intake } \\
\text { during } \\
\text { lactation. }\end{array}$ & $\begin{array}{l}\text { Prospective } \\
\text { cohort clinical } \\
\text { study. }\end{array}$ & $\begin{array}{c}n=16 \\
\text { breastmilk } \\
\text { samples, } 1 \text { per } \\
\text { mother } \\
\text { (healthy Euro- } \\
\text { Canadian } \\
\text { mothers, } \\
\text { divided into } \\
\text { two groups } \\
\text { classified as } \\
\text { milk- or } \\
\text { almond } \\
\text { beverage- } \\
\text { consumers). }\end{array}$ & $\begin{array}{c}\text { Self- } \\
\text { Administered } \\
\text { 24-h (ASA24) } \\
\text { diet-recall } \\
\text { survey for the } \\
\text { 24-h period } \\
\text { preceding milk } \\
\text { collection. }\end{array}$ & $\begin{array}{l}\text { At } 5 \text { months } \\
\text { postpartum, } \\
\text { manual } \\
\text { expression of a } \\
\text { few drops of } \\
\text { milk } \\
\text { (discarded) } \\
\text { before } \\
\text { collecting } 10 \\
\mathrm{~mL} \text { of foremilk } \\
\text { in the morning. }\end{array}$ & $\begin{array}{l}93 \text { (median = } \\
\text { 87) HMOs. } \\
\text { Quantitation of } \\
\text { total reducing } \\
\text { sugars, } \\
\text { Neu5Ac and } \\
\text { Neu5Ac, } \\
\text { neutral } \\
\text { monosaccha- } \\
\text { ride analysis } \\
\text { (fucose, } \\
\text { galactose), } \\
\text { HMO profiling } \\
\text { by CE-LIF, and } \\
\text { targeted HMO } \\
\text { analysis by } \\
\text { HPLC-MS. } \\
\text { Determination } \\
\text { of secretor } \\
\text { status by CE of } \\
\text { the median } \\
\text { levels of 2'-FL, } \\
\text { LDFT, and } \\
\text { LNFP I. }\end{array}$ & $\begin{array}{l}\text { In Se+ samples } \\
(n=12), \\
\text { relative levels } \\
\text { of Fuc and Gal } \\
\text { in HMOs were } \\
\text { positively } \\
\text { correlated with } \\
\text { both the total } \\
\text { sugars ( } p< \\
\text { 0.01) and total } \\
\text { dietary fiber } \\
(p<0.05) \\
\text { ingested } \\
\text { within the 24-h } \\
\text { period prior to } \\
\text { milk collection. } \\
\text { Several } \\
\text { sulfonated/ } \\
\text { phosphory- } \\
\text { lated HMOs } \\
\text { were positively } \\
\text { correlated with } \\
\text { breast milk mo- } \\
\text { nounsaturated } \\
\text { and polyunsat- } \\
\text { urated fats, } \\
\text { and negatively } \\
\text { correlated with } \\
\text { levels of } \\
\text { saturated fats } \\
\text { ( } p<0.05) .\end{array}$ \\
\hline $\begin{array}{l}\text { Samuel et al. } \\
{[8]}\end{array}$ & $\begin{array}{c}\text { Maternal } \\
\text { pre-pregnancy } \\
\text { body } \\
\text { composition } \\
\text { (compared the } \\
\text { concentrations } \\
\text { of HMOs } \\
\text { between } \\
\text { overweight } \\
\text { normal weight } \\
\text { women at six } \\
\text { different time } \\
\text { points over the } \\
\text { first four } \\
\text { months of } \\
\text { lactation, } \\
\text { adjusted for } \\
\text { milk group). }\end{array}$ & $\begin{array}{l}\text { Longitudinal, } \\
\text { observational, } \\
\text { multicenter } \\
\text { European } \\
\text { study (Atlas of } \\
\text { Human Milk } \\
\text { Nutrients). }\end{array}$ & $\begin{array}{c}n=1491 \text { milk } \\
\text { samples over } 6 \\
\text { visits from } 290 \\
\text { women } \\
\text { (healthy } \\
\text { lactating } \\
\text { European } \\
\text { women from } \\
\text { France, Italy, } \\
\text { Spain, } \\
\text { Romania, } \\
\text { Portugal, } \\
\text { Sweden, and } \\
\text { Norway). }\end{array}$ & $\begin{array}{l}\text { Self-reported } \\
\text { maternal } \\
\text { pre-pregnancy } \\
\text { weight and } \\
\text { height to } \\
\text { calculate } \\
\text { pre-pregnancy } \\
\text { BMI } \\
\text { (categorized as } \\
\text { normal weight: } \\
18.5-24.9 \\
\text { kg/m2 and } \\
\text { overweight: } \\
25.0-29.9 \\
\text { kg/m2). } \\
\text { Weight loss } \\
\text { postpartum } \\
\text { (kg). }\end{array}$ & $\begin{array}{c}\text { Samples } \\
\text { collected at } 3 \\
\text { days, } 14 \text { days, } \\
1 \text { month, } 2 \\
\text { months, } 3 \\
\text { months and } 4 \\
\text { months after } \\
\text { delivery. Milk } \\
\text { collected at } \\
\text { 11h00 } \pm 2 \text { h00, } \\
\text { electric breast } \\
\text { pump, single } \\
\text { (same) breast } \\
\text { for the entire } \\
\text { study (emptied } \\
\text { in the previous } \\
\text { feed), mixed } \\
\text { full breast } \\
\text { expression. }\end{array}$ & $\begin{array}{l}\text { Targeted HMO } \\
\text { analysis by } \\
\text { HPLC-MS, and } \\
\text { profiling of } 20 \\
\text { HMOs by } \\
\text { CE-LIF. } \\
\text { Summed total } \\
\text { amount of } \\
\text { HMOs } \\
\text { between milk } \\
\text { groups, } \\
\text { categorized } \\
\text { mothers in one } \\
\text { of four groups } \\
\text { based on } \\
\text { presence of } \\
\text { specific } \alpha-1,2 \\
\text { and } \alpha-1,4- \\
\text { fucosylated } \\
\text { HMOs }\left(2^{\prime}-\mathrm{FL},\right. \\
\text { and LNFP II). }\end{array}$ & $\begin{array}{c}\text { Overweight } \\
\text { women (BMI } \\
25.0-29.9 \\
\text { kg/m2) had } \\
\text { higher } \\
\text { concentrations } \\
\text { of } 3^{\prime} \mathrm{SL}, 6^{\prime} \mathrm{GL} \\
(p<0.05) \text {. The } \\
\text { magnitude of } \\
\text { the effect } \\
\text { observed were } \\
\text { generally low, } \\
\text { and for } 3^{\prime} \mathrm{SL} \\
\text { and 6'GL } 22 \\
\text { and } 29 \text { mg/L, } \\
\text { respectively. }\end{array}$ \\
\hline
\end{tabular}


Table 1. Cont.

\begin{tabular}{|c|c|c|c|c|c|c|c|}
\hline Reference & $\begin{array}{l}\text { Exposure } \\
\text { Variables of } \\
\text { Interest }\end{array}$ & Study Design & $\begin{array}{c}\text { Population } \\
\text { and Sample } \\
\text { Number }\end{array}$ & $\begin{array}{c}\text { Maternal } \\
\text { Nutritional } \\
\text { Assessment } \\
\text { Tool }\end{array}$ & $\begin{array}{c}\text { Milk } \\
\text { Sampling }\end{array}$ & $\begin{array}{c}\text { HMO } \\
\text { Analysis }\end{array}$ & $\begin{array}{c}\text { Relevant } \\
\text { Main } \\
\text { Outcomes }\end{array}$ \\
\hline $\begin{array}{c}\text { Seppo et al. } \\
{[22]}\end{array}$ & $\begin{array}{c}\text { Maternal } \\
\text { probiotic sup- } \\
\text { plementation } \\
\text { during } \\
\text { pregnancy } \\
\left(5 \times 10^{9} \text { CFU }\right. \\
\text { Lactobacillus } \\
\text { rhamnosus GG, } \\
5 \times 10^{9} \text { CFU L. } \\
\text { rhamnosus } \\
\text { LC705, } 2 \times 10^{8} \\
\text { CFU Bifidobac- } \\
\text { terium breve } \\
\text { Bb99, } 2 \times 10^{9} \\
\text { CFU Propioni- } \\
\text { bacterium } \\
\text { freudenreichii } \\
\text { ssp. shermanii } \\
\text { JS as } \\
\text { freeze-dried } \\
\text { capsules twice } \\
\text { daily). }\end{array}$ & $\begin{array}{l}\text { Randomized, } \\
\text { double-blind, } \\
\text { placebo- } \\
\text { controlled } \\
\text { study with } 2 \\
\text { parallel groups } \\
\text { (probiotic } \\
\text { preparation or } \\
\text { a placebo for } 2 \\
\text { to } 4 \text { weeks } \\
\text { before delivery, } \\
\text { i.e., from } 36 \\
\text { weeks' } \\
\text { gestation until } \\
\text { the birth). }\end{array}$ & $\begin{array}{c}n=81 \\
\text { colostrum } \\
\text { samples } \\
\text { (pregnant } \\
\text { women } \\
\text { carrying } \\
\text { children at } \\
\text { increased risk } \\
\text { for allergy } \\
\text { from the } \\
\text { Helsinki } \\
\text { suburban } \\
\text { area). }\end{array}$ & $\begin{array}{l}\text { Developed } \\
\text { questionnaire } \\
\text { to assess } \\
\text { compliance to } \\
\text { the supplemen- } \\
\text { tation. }\end{array}$ & $\begin{array}{l}\text { Stored frozen } \\
\text { colostrum } \\
\text { samples from a } \\
\text { previous RCT } \\
\text { of probiotic } \\
\text { supplementa- } \\
\text { tion study of } \\
1223 \text { pregnant } \\
\text { women. }\end{array}$ & $\begin{array}{c}\text { HPLC; } 19 \\
\text { HMOs. } \\
\text { Freezing does } \\
\text { not affect } \\
\text { HMO levels. }\end{array}$ & $\begin{array}{l}\text { 3FL and } 3^{\prime} \text { SL } \\
\text { significantly } \\
\text { higher in the } \\
\text { probiotic group } \\
\text { ( } p=0.008 \text { and } p \\
=0.006) . \\
\text { Levels of } \\
\text { DFLNH, LNnT, } \\
\text { LNFP, } 6^{\prime}-S L \\
\text { were lower in } \\
\text { the supplemen- } \\
\text { tation group } \\
(p=0.005, p= \\
0.01, p=0.03, p \\
=0.03) \text {. These } \\
\text { changes are } \\
\text { consistent with } \\
\text { a change in } \\
\text { select } \\
\text { pathways in } \\
\text { overall HMO } \\
\text { biosynthesis. }\end{array}$ \\
\hline $\begin{array}{l}\text { Tonon et al. } \\
\text { [30] }\end{array}$ & $\begin{array}{l}\text { Maternal } \\
\text { anthropometry } \\
\text { (pre- and post- } \\
\text { pregnancy } \\
\text { BMI) and } \\
\text { allergic disease } \\
\text { status. }\end{array}$ & $\begin{array}{l}\text { Cross- } \\
\text { sectional, } \\
\text { observational } \\
\text { study. }\end{array}$ & $\begin{array}{c}n=78 \text { mature } \\
\text { human milk } \\
\text { samples, } 1 \text { per } \\
\text { mother (Por- } \\
\text { tuguese/Brazilian } \\
\text { women, } \\
\text { multi-ethnic) }\end{array}$ & $\begin{array}{c}\text { Maternal } \\
\text { pre-pregnancy } \\
\text { BMI } \\
\text { (self-reported } \\
\text { weight/obtained } \\
\text { from medical } \\
\text { records); BMI } \\
\text { during } \\
\text { lactation } \\
\text { (weight and } \\
\text { height } \\
\text { measured at } \\
\text { inclusion, } \\
\text { median: } 32 \\
\text { days, IQR: } \\
\text { 25-46 days } \\
\text { postpartum); } \\
\text { allergic disease } \\
\text { by ISAAC } \\
\text { questionnaire. }\end{array}$ & $\begin{array}{c}\text { Manual } \\
\text { expression of } \\
\text { the breast } \\
\text { opposite to the } \\
\text { one previously } \\
\text { emptied by the } \\
\text { infant; } 5-15 \mathrm{~mL} \\
\text { collected in a } \\
\text { sterilized glass } \\
\text { bottle in the } \\
\text { morning } \\
\text { (8:30-12:00 } \\
\text { am), at } 17 \text { to } 76 \\
\text { days } \\
\text { postpartum } \\
\text { (median: } 32 \\
\text { days, IQR: } \\
\text { 25-46 days)). }\end{array}$ & $\begin{array}{c}\text { LC-MS, } \\
\text { performed in } \\
\text { duplicate; } 16 \\
\text { HMOs (to } \\
\text { represent } \\
\text { about } 90 \% \text { of } \\
\text { the total HMOs } \\
\text { in human } \\
\text { milk). Secretor } \\
\text { and Lewis } \\
\text { phenotype of } \\
\text { the mothers } \\
\text { based on the } \\
\text { presence of } \\
\text { indicative } \alpha 1-2 \\
\text { and } \alpha 1-4 \\
\text { fucosylated } \\
\text { HMOs. }\end{array}$ & $\begin{array}{l}\text { Maternal body } \\
\text { composition } \\
\text { during } \\
\text { lactation is } \\
\text { associated with } \\
\text { concentrations } \\
\text { of some HMOs } \\
\text { in Se+ women } \\
\text { (positive } \\
\text { correlation } \\
\text { between } 2^{\prime}-\mathrm{FL} \\
\text { and maternal } \\
\text { BMI }(\mathrm{r}=0.30)) .\end{array}$ \\
\hline
\end{tabular}

FFQ, food frequency questionnaire; BMI, body mass index; HMOs, Human Milk Oligosaccharides; FUT2, Galactoside 2-alpha-Lfucosyl transferase 2; 2'-FL, 2'-Fucosyllactose; HPLC, high-performance liquid chromatography; LC-GCMS, liquid chromatography-gas chromatography-mass spectrometry; HPLC-FLD, Fluorescence detector-high performance liquid chromatography; MS, mass spectrometry; CE-LIF, Capillary electrophoresis with laser-induced fluorescence detection; Nano-HPLC-Chip/TOF MS, Nano- high-performance liquid chromatography-Chip/Time of Flight mass spectrometry; LNFP, Lacto-N-fucopentaose; LNnT, Lacto-N-Neotetraose; FM, fat mass; FFM, fat-free mass; 6'-SL, 6'Sialyllactose; LSTb, sialyl-lacto-N-tetraose.

\subsection{Quality of Reporting Assessment}

In order to appraise the methodological quality of the studies, the Critical Appraisal Skills Programme (CASP) qualitative checklist tools were used, as endorsed by the Cochrane Qualitative and Implementation Methods Group (https: / / casp-uk.net/casp-tools-checklists/, accessed on 5 September 2020). Twelve studies were assessed using the Quality Assessment Tool for Observational Cohort and Cross-Sectional Studies, and two were assessed using the Quality Assessment of Controlled Intervention Studies tool [31]. Two reviewers (C.B. and J.M.) scored each manuscript independently and discussed results. A study was deemed to be of "good" (10-14 points), "fair" (5-9 points), or "poor" (<5 points) quality, based upon the validity of results and risk of bias. Critique ratings indicated that even in the case of "fair" 
scores, study results were valid despite being susceptible to some bias. We did not exclude studies based on quality as we aimed to canvas all results available for the scoping review.

\section{Results}

\subsection{Synthesis}

The search strategy identified a total of 2861 records (Figure 1). Three additional articles were identified from the reference lists of included publications. After removal of duplicates, 2137 remained. Based on title and abstract screening, 1698 articles were excluded. Of the remaining articles, 33 met the stated inclusion criteria and were subjected to full-text assessment. In total, 14 publications were included in the scoping review, with data from 1388 lactating women and 4011 milk samples. A summary evidence table of included studies is provided as Table 1 . Papers reported on maternal dietary intake $(n=3)$, dietary supplementation interventions $(n=2)$, and maternal body composition $(n=9)$, in relation to HMO composition of milk samples. The articles reflect a quite recent interest in the link between maternal factors and HMO composition, being published between 2013 and 2020. Four studies were conducted North America [12,27-29], two in South America [15,30], three in Europe [8,19,22], two in Africa [14,18], one in Australia [16], and one in Asia [21]. One large multisite study with 11 international cohorts was also included [20].

\subsection{Analysis of Methodologies}

\subsubsection{Study Design and Sample Characteristics}

Of the 14 studies included in the analysis, four were cross-sectional $[20,21,29,30]$ and five were prospective observational $[12,14,19,27,28]$ studies. Most studies investigated a range of variables, including maternal nutrition factors and their associations with $\mathrm{HMO}$ concentrations in the samples. Three studies were classified as prospective longitudinal studies with HMO-related outcomes assessed three to six times over the postpartum period. Changes in HMO profiles over time could therefore be reported upon $[8,15,16]$. Two studies were controlled experiments: a single (assessor)-blind, parallel group controlled multinutrient supplementation trial [18], and a double-blind, placebo-controlled study with two parallel groups investigating maternal probiotic supplementation [22]. Seven of the studies (50\%) were deemed to be of good quality [12,18-20,22,28,30] and half received a "fair" rating [8,14-16,21,29,30]. Both controlled intervention studies were rated highly, with adequate randomisation and intention-to-treat analyses [18,22]. The sample size varied substantially between studies, ranging from pilot/proof of concept $(n=20$ mother-infant pairs) [16] to a large multi-national trial ( $n=410$ across 11 sites) [20]. The largest study with a sample size of $n=427$ women [12] was embedded within a larger observational study, the Canadian Healthy Infant Longitudinal Development (CHILD) cohort which recruited 3624 pregnant women [32]. Typically, the sample size was pragmatically chosen and many of the studies investigating dietary parameters and HMOs did not report on the strength of correlational relationships. Since work in this field is still very much exploratory in nature, the level of variance of interest from a clinical perspective remains uncertain. Most of the studies considered basic exposure variables such as: maternal age, ethnicity, socioeconomic status, geographical location, health status, gestational age, parity, birth weight, mode of delivery, and breastfeeding status. Few studies $(n=3)$ investigated the use of nutritional supplements (including herbal and pre-/probiotics), and only one study used body composition indices other than the standard calculation of body mass index (BMI) [16].

\subsubsection{Human Milk Sampling Procedures}

Milk sampling protocols varied between studies, reflecting the range of methodologies applied in the field. Most studies $(n=11)$ measured the HMO composition in mature milk samples However, one study measured HMOs in stored frozen colostrum samples (0 to 4 days postpartum) [22] and two studies included samples of transitional milk at 
28 days [8] and 3 days postpartum [15]. To assess trajectories and account for the fact that HMO profiles vary throughout the course of lactation [9], some studies sampled milk at multiple time points $[14-16,19,20,28]$, whilst others only included a single sample of mature milk in their design $[12,18,21,27,29]$. Of the papers that reported the time of day of sampling, most indicated this was done either in the morning $[14,15,27,30]$ or around midday $[8,28]$. Only four studies reported on HMO composition in combined fore- and hind-milk from multiple feeds over $24 \mathrm{~h}[12,29]$ or pooled samples of both breasts [14,19]. Substantial sources of variance are evident across the studies with regards to breast milk sampling and handling protocols, time of day of expression, partial vs full breast expression, and the subsequent storage of samples prior to analysis. While evidence suggests that the HMO composition in mature milk is not affected by cold storage and seems to remain stable over and between days [33], the effect of long-term storage and multiple handling procedures on HMO composition remains unknown [1].

\subsubsection{Human Milk Oligosaccharides Analysis}

For the analysis and profiling of HMOs, a variety of chromatographic, electrophoretic, and spectrometric approaches were used. Most of the studies used quantification methods based on liquid chromatography (LC) coupled to mass spectrometric (MS) detection $[12,14,18-20,22,29,30]$. Two studies used capillary electrophoresis for HMO profiling in addition to this $[8,27]$. A further two utilised high performance LC (HPLC) with fluorescence detection [15,21], and one used LC-GCMS (liquid and gas chromatography) for untargeted metabolomics analysis [28]. The impact of varying $\mathrm{HMO}$ analysis methods on study outcomes is difficult to ascertain; this would require the analysis of the same samples across different laboratories and methods for assessment of variance. There is currently no gold standard method for $\mathrm{HMO}$ analysis, and variances across studies and laboratories remain.

Most of the studies sought to quantify at least 16-20 individual HMOs that typically account for $>90 \%$ of total HMO content [12], and their concentrations were summed to estimate total HMO concentrations $[8,12,14,15,19,20,22,29,30]$. The following HMOs were often summed to provide an estimate of total HMO content in milk samples: 2'fucosyllactose (2'-FL), 3-fucosyllactose (3FL), $3^{\prime}$-Sialyllactose ( $\left.3^{\prime} \mathrm{SL}\right), 6^{\prime}$-Sialyllactose (6'SL), lacto-N-tetraose (LNT), lacto-N-neotetraose (LNnT), lacto-N-fucopentaose I/II/III (LNFP I, LNFP II, LNFP III), sialyl-LNT (LST) b, LSTc, difucosyl-LNT (DFLNT), disialyl-LNT (DSLNT), fucosyl-lacto-N-hexaose (FLNH), difucosyl-lacto-N-hexaose (DFLNH), fucosyldisialyl-lacto-N-hexaose (FDSLNH), disialyl-lacto-N-hexaose (DSLNH), difucosyllactose (DFLac), and lacto-N-hexaose (LNH). Two studies did not report on HMO quantification as such, and instead reported on a calculated total HMO value estimated form the total milk carbohydrate and lactose content [16], and on milk sialic acid concentrations as a proxy for sialylated HMO content [21]. HMO concentrations are strongly influenced by genetics [12], thus most of the studies assessed maternal Secretor status as a confounding variable $[8,12,14,15,19,20,22,27,29,30]$. Secretor can be determined by genotyping the presence of FUT2 genes via polymerase chain reaction-random fragment length polymorphisms [34]. However, studies in this review used a proxy method involving HMO identification and the presence or abundance of fucosylated HMOs such as $2^{\prime}$-FL and LNFP I $[12,15,19,20,29,30]$. Davis et al. chose a relative cutoff of $>6 \%$ relative $\alpha(1-2)$ fucosylation to be indicative of Secretor status [14]. Only one paper reported both Secretor and Lewis phenotypes based on the abundance or lack of $\alpha 1-2$ and $\alpha 1-4$ fucosylated HMOs [31].

\subsubsection{Nutrition Assessment}

Dietary intake assessment methods. Maternal dietary intake was assessed directly in three studies $[12,21,27]$. Validated food frequency questionnaires (FFQ) were used in two studies $[12,21]$, where the usual frequency of consumption of foods and beverage were assessed retrospectively. Azad et al. [12] used the Women's Health Initiative (USA) FFQ during late pregnancy (not during lactation) and assessed pre-pregnancy BMI using self- 
reported weight. This FFQ is validated in non-pregnant women, captures usual dietary intake, and was used to calculate the Healthy Eating Index (HEI) score, resulting in an overall score that represents the quality of the whole diet as compared to recommendations of the Dietary Guidelines for Americans [12]. A more recent study by Quin et al. [27] utilised the Self-Administered 24 h (ASA24) Dietary Assessment tool [35], which was completed by 16 lactating women in the $24-\mathrm{h}$ prior to milk sample collection. This meant that the nutrition assessment was based on the mother's dietary intake immediately prior to milk expression during lactation. In contrast, the FFQ tool utilised by Azad et al. [12] assessed longer term or usual maternal dietary intake during pregnancy. Both studies [12,30] represented dietary intake as food groups and reported on macronutrient profiles (total energy, fat, protein, and carbohydrates). The third study by Qiao et al. [21] also used an FFQ that included selected micronutrients (vitamins A, E, C, calcium, zinc, manganese, iron, and selenium). This study sought to investigate the impact of maternal diet on breastmilk components, specifically sialic acid. HMOs were not quantified; rather breastmilk sialic acid levels (of which $82 \%$ was bound to oligosaccharides) were measured, and the 72-h FFQ was performed during lactation (40 \pm 7 days postpartum). This FFQ assessed participants' recent dietary intake relative to milk sampling (single occurrence) and food models were used to improve the accuracy of estimation of dietary intake [21]. Other dietary exposures or patterns were not explored in these three studies. Dietary intake was not directly measured in a probiotic supplementation trial [22] and a multi-nutrient supplement trial [18]. In these intervention studies, adherence to protocol indicating actual dietary intake of supplements was assessed using developed questionnaires.

Anthropometric assessment methods. Eleven of the fourteen studies assessed maternal anthropometric characteristics, including BMI, gestational weight gain, and body composition. These anthropometric measures can be used as a proxy or indicator of both short- and longer-term nutritional status. Basic maternal anthropometric measures of height, body weight/mass and BMI were assessed in 11 of the studies [8,12,14-16,18-20,28-30]. BMI was calculated and classified using the standard equation: Weight/Height ${ }^{2}$ (Quetelet's index; $\mathrm{kg} / \mathrm{m}^{2}$ ) [36]. However, BMI is a crude measure and cannot be used to distinguish between fat and lean mass [37]. Five studies assessed both pre-pregnancy BMI and BMI during lactation $[8,18,19,28,30]$, though key confounders of BMI such as levels of physical activity, dietary intake and hormonal influences were not accounted for. Gestational weight gain (GWG) was determined in four studies as the difference between body weight at the final prenatal visit $[15,19,28]$ and pre-pregnancy weight (self-reported or from medical records). Only Gridneva et al. [38] used bioelectrical impedance spectroscopy (BIS) on four occasions $(2,5,9$, and/or 12 months postpartum) in addition to body weight and BMI to assess maternal body composition during lactation. BIS is considered a more robust technique of assessing body composition than BMI.

\subsection{Maternal Diet Quality and Dietary Components}

When overall pregnancy diet quality was assessed by Azad et al. [12] in their large general population-based cohort study $(n=427)$ no associations with HMO concentrations in milk collected at 3-4 months post-partum were found. However, total energy intake, although not associated with overall HMO concentrations, was positively correlated with some individual HMOs (LNTand DFLNT) (all $\rho=-0.11$ to +0.1$)$ (12). Davis et al. [14] found that in the so-called 'dry' season in African Gambia, when food is more plentiful, mothers could produce significantly more HMOs. Season was described as a proxy for dietary energy intake. It was thought that as total dietary energy intake may be greater in the dry season, the overall milk output may be increased. However, no direct assessments were made of the mothers' dietary intake so the link between HMO composition and seasonal environmental changes as a proxy for dietary energy intake in this study remains speculative. Azad et al. [12] also reported on seasonal variation in some HMOs but suggested that environmental factors such as climate or exposure to sunlight or allergens might influence HMO concentrations more than dietary energy intake. 
Variable associations between maternal dietary macronutrient intakes and HMO concentrations were found in three studies [12,21,27]. Azad et al. [12] found weak negative correlations between total protein, empty calories, and sialyl-lacto-N-tetraose b (LSTb). Quin et al. [27] also reported that some maternal dietary components could influence the biosynthesis of HMOs during lactation, particularly in Secretor-positive women. Several sulfonated HMOs were positively correlated with monounsaturated and polyunsaturated fats, and negatively correlated with levels of saturated fats and dietary cholesterol $(p<0.05)$. Both studies also explored the potential association between food groups and HMO composition in the milk samples. Quin et al. [27] found that only fruit, a dietary source of simple sugars and dietary fiber, was positively correlated with absolute amounts of selected HMOs and with levels of galactose and fucose present in HMOs, while sialic acid levels were significantly lower $(p<0.05)$ [30]. On the other hand, Azad et al. [12] found a positive correlation between wholegrains and fucosyllacto- $N$-hexaose. No other significant relationships between food groups or dietary patterns and HMO concentrations were reported.

In terms of micronutrient content of the maternal diet, evidence is also limited at this stage. In the study by Qiao et al. [21], mothers who had higher dietary vitamin A intakes (from food sources; $602.22 \pm 126.46 \mu \mathrm{g}$ /day) had a significantly higher concentration of sialic acid in their milk samples $(p=0.000)$. No significant associations for any other dietary components were noted, although profiling individual HMOs at one time point only is a limitation of the cross-sectional method used. Repeating dietary assessment over time may give more insight into the longitudinal changes in HMO profiles over the course of lactation. Three of the studies investigated associations between dietary supplementation (nutrient or probiotic) and HMO composition [12,18,22]. Azad et al. found that only DSLNH was notably higher in the breast milk of mothers who self-reported taking multivitamins supplements [12]. In a large-scale interventional trial, Jorgensen et al. [18] hypothesised that nutrient supplementation during late pregnancy would impact $\mathrm{HMO}$ levels, presumably secondary to improving overall nutritional status. However, when assessing a cohort of mothers in Africa, most of whom had suboptimal nutritional intake, they found no effect of a lipid-based nutrient supplements on HMO levels $(p>0.10$ for all comparisons) [18]. Pregnant women $(n=647)$ were given either micronutrient capsules containing 18 micronutrients or a $20 \mathrm{~g}$-dose of a high-energy, micronutrient fortified lipidbased nutrient supplement with the same 18 micronutrients and 4 additional minerals, $2.6 \mathrm{~g}$ protein, and $10 \mathrm{~g}$ fat to provide $118 \mathrm{kcal}$ of total energy. Neither intervention resulted in increased HMO levels at 6 months postpartum. However, no investigation was made into the adequacy or quality of the mothers' diet, so other dietary influences could not be discounted. Since participants in this study were often of a lower socioeconomic status and with low BMIs, it is possible that the effects of additional nutrient supplementation may only be seen in energy-replete, well-nourished individuals.

One study reported on the impact of probiotic supplementation on HMO profiles [22], however their results reported on a complex interplay between the maternal microbiome, the milk microbiome, and HMOs, which made it difficult to report directly on the relationship between probiotic supplementation and HMOs. In this randomized, double-blind, placebo-controlled study probiotic supplements were given to mothers $(n=81)$ during the late stages of pregnancy. The mothers in the probiotic group took twice daily capsules containing Lactobacillus rhamnosus GG, Lactobacillus rhamnosus LC705, Bifidobacterium breve Bb99, and Propionibacterium freudenreichii ssp. shermanii JS. Their newborn infants received an opened capsule containing the same probiotics mixed with 20 drops of sugar syrup containing $0.8 \mathrm{~g}$ of galacto-oligsaccharides once daily for 6 months after birth. Concentrations of 3-fucosyllactose (3FL) and $3^{\prime}$-sialyllactose (3'SL) in colostrum from the mothers in the probiotic supplementation group increased significantly compared with those in the placebo group ( $p=0.008$ and $p=0.006$, respectively). An opposing and significant decrease in 6 -sialylated HMOs was also noted ( $p<0.05$ for all). It is not clear whether the changes in 3FL and $3^{\prime}$ SL concentrations were specific to the types of probiotics used in this study, but it does suggest that external factors can manipulate HMO profiles. The investigators 
further suggested that probiotic supplements could influence the amount or activity of enzymes that are functional in specific biological pathways [22].

Overall, studies assessing maternal dietary intake or supplementation and the association with $\mathrm{HMO}$ volume and profiles are scarce. The variance in the methods of assessment is too great to allow for general conclusions. Dietary intake and quality in pregnancy does not appear to be of notable influence [12], whereas some individual dietary components and supplements may be influential in the short term or during the postpartum period $[12,21,22,27]$. The majority of the included studies had small samples which also limits the ability to draw any significant conclusions. Assessment of lactational diet through multiple dietary recalls may assist in improving validity of dietary methods. In addition, other potentially more robust methods for exploring diet quality and dietary patterns through principal component analyses and other similar methods, have not been explored.

\subsection{Body Composition Assessment}

A number of measures performed at various times in relation to lactation and considering different genetic subgroups exist in the literature examining maternal anthropometry and HMO composition. When assessing pre-pregnancy BMI and maternal body weight, Ferreira et al. [15] found that both measures were moderately correlated with LNnT (Spearman rank correlation 0.4) but Samuel et al. [8] found that a higher BMI was associated with a lower concentration of this same HMO and some others (LNT and LNFP V; all $p<0.05$ ). The studies were however performed on different cohorts (South American versus European) and the substantial influence of genetic variation or geographic location may have outweighed the influence of maternal physiological status. The association noted in the latter study was also inconsequential after statistical correction for multiple testing, and then only $3^{\prime} \mathrm{SL}$ and $6^{\prime} \mathrm{GL}$ remained positively correlated with a higher BMI category over the first four months of lactation $(p<0.05)$ [8]. Both Secretor and non-secretor mothers were included in the analysis in these studies. Larsson et al. [19] found no associations between HMOs and pre- or post-pregnancy BMI, nor with gestational weight gain in Secretor positive women. However, when combining Secretors and non-secretors, maternal pre-pregnancy BMI was weakly associated with some individual HMO values. These same associations became significant with BMI measured at 5 months postpartum (i.e., negative associations with $6^{\prime}$-SL and LSTb $(p=0.03)$, positively with $2^{\prime}$-FL $(p<0.05$ for all). Total HMO volume and total HMO-bound fucose were also positively associated with postpartum BMI ( $p=0.015$ and $p=0.033$, respectively). The timing of anthropometric measurements relative to pregnancy and lactation appears to be of consequence. Another example of this impression is LNFP III: one study reported a moderately negative correlation for this HMO with pre-pregnancy body weight $(r=-0.04)$ [15], another reported a positive correlation $(r=0.20)$ with postpartum body weight [20].

The association between anthropometric measures and individual HMOs appears to be more consistent when only considering postpartum assessments. McGuire et al. [20] performed all measurements during the lactational period and found correlations between maternal weight, BMI, and individual HMOs (positive correlations with $2^{\prime}-\mathrm{FL}(\mathrm{r}=0.20$ for both) and FLNH $(r=0.19$ and 0.15 , respectively), and inverse correlations with DSLNT $(r=20.20$ and 20.24) and LNnT $(r=20.16$ and 20.21). Likewise, Tonon et al. [31] measured maternal BMI in the early postpartum period (17 to 76 days, median: 32 days, IQR: $25-46$ days), and found a positive correlation with $2^{\prime}-\mathrm{FL}(\mathrm{r}=0.30, p<0.05)$. As this was a cross-sectional study, the persistence of the association over time could not be determined. At one month postpartum, Isganaitis et al. [28] found that maternal BMI was significantly associated with 2'-FL, LNFP I, and LNFP II/III ( $p<0.05$ for all), but these associations did not extend to six months postpartum.

Overall, more evidence is required to confirm the associations between maternal anthropometrics and singular HMOs. Perhaps the most evident association across studies and populations is indeed for 2'-FL positively correlating with BMI in the postpartum period $[19,20,28,30]$. Future studies would do well to separate results for Secretor and non- 
secretor women. Further, body weight and BMI are not good indicators of adiposity during the postpartum period [20] and other more valid techniques should be used to investigate the associations more definitively. Gridneva et al. [16] used BIS on four occasions (2, 5, 9, and/or 12 months postpartum) in addition to maternal body weight and BMI measures. They found no associations with maternal body composition (fat mass, fat-free mass, percentage fat mass, and height-normalized indices of fat mass and fat-free mass) and calculated HMO levels $(n=20)$. The authors did not quantitate individual HMOs and perhaps direct identification of HMOs might have better revealed any associations between indices of body composition and HMO profiles during lactation.

\section{Discussion}

The aim of this scoping review was to investigate any known associations between maternal nutritional intake and patterns, as well as nutritional status and the volume and/or composition of HMOs in human milk. We also sought to investigate hypotheses around possible mechanisms by which maternal diet and related factors may influence HMO profiles. Work in this field is still in the initial exploratory stages, with most publications emerging in the last three years. Following the PRISMA-ScR guidelines and a systematic search of the literature, fourteen studies met the inclusion criteria. Only three reported on maternal dietary intake, nine on maternal body composition, and two on controlled dietary supplementation interventions. In total, data from 1388 lactating mothers and 4011 milk samples were included. The review identified singular associations between the HMO composition of human milk and components of the maternal diet or body composition measures.

Variation was introduced via interlaboratory differences in the protocols and procedures utilised for milk sampling and HMO analyses, which could impact the HMO quantities and profiles reported. However, most studies included in this review used chromatographic and mass spectrometric methods consistent with current practices to identify and quantify a select group of more abundant HMOs. The existence of published data and analytical standards was likely to also impact on the choice of HMOs examined $[9,30]$. Several studies focused on quantifying less than $20 \%$ of more than 200 structurally distinct and known HMOs [39] and accounted for the major source of interindividual variation in HMO levels; that is the effects of polymorphism of the Secretor and Lewis genes [2,11,30]. Results should be reported separately for Secretor and non-secretor women, although there is notable variability in HMO profiles even within the same phenotypes [30]. Discrepancies exist in the literature as to whether differences in maternal secretor status affect infant outcomes: evidence suggests no effect on growth outcomes [30], yet some effects on infant microbiota [40]. Future studies should also calculate the absolute amount of milk (and therefore HMOs) ingested by the infant, as certain health outcomes, such as growth rate, appear to be related to the volume of breastmilk consumed [16].

Research into the influence of maternal dietary intake on HMO composition and profiles in colostrum and mature milk is in its infancy and has been explored in only three studies included in this review [12,21,27]. Significant variability exists in dietary intake assessment methods, as reported above. Diet as an exposure is difficult to measure, due in part to the complexity and number of dietary elements that must be accounted for. Although diet quality has been assessed using a robust measure (HEI) [12], other methods for assessing diet quality such as dietary pattern analyses, could be used to further explore relationships between diet and HMOs. Further, diet quality was only assessed during pregnancy and should be assessed during lactation, preferably at multiple timepoints, as it is likely that maternal diet changes post pregnancy. The literature points to a decrease in overall diet quality (along with fruit and vegetable intake), and an increase in the intake of energy-dense yet nutrient-poor food choices post pregnancy [41].

The fact that HMO profiles also varies across lactation gives further reason to perform multiple dietary assessments in the postpartum period. Multiple 24-h dietary recalls may be more valid and reliable and provide an acceptable and more practical alternative to a 
weighed food diary. Quin et al. [27] used a single dietary recall in the postpartum period, in a sample too small to assess diet quality. As such their work was centered on dietary components alone but did reveal correlations between diet (total sugars, dietary fibre) and the fucose/galactose in HMOs. They suggest maternal diet is important in the biosynthesis of HMOs, which is likely since the biosynthetic pathway initiates from activated monosaccharides. This suggestion may be echoed in very recent work by Seferovic et al. [42] who found that changing maternal dietary energy and in particular, carbohydrate sources, resulted in alterations in some of the major HMO constituents in a short duration of time. In a cross-over controlled feeding study ( $n=7$ lactating women), a high fat diet (contributing $>40 \%$ total energy) resulted in a decrease in the concentration of sialylated HMOs, and a higher glucose versus galactose diet affected the profiles of fucosylated HMOs. These studies are expensive to implement and have limited generalisability, partly due to the requirement that participants receive controlled diets but do reveal mechanistic pathways in a clinical sense. Larger, longer duration intervention studies may reveal additional dietary components that impact the HMO composition of milk, as well as provide further evidence in identifying the underlying mechanisms driving such changes. However, these types of studies do need to be complemented with population-based studies to generate practical dietary advice to lactating women. Presently, the relationship between dietary manipulations and HMOs remains speculative due to the lack of a clear understanding of the pathways of HMO biosynthesis and because human milk supplies infant needs in a complex and dynamic manner. This review found no consistent relationships between HMOs and maternal dietary exposures in general populations. In fact, it is generally accepted that gross milk composition may be safeguarded against moderate variations in maternal nutritional status [43] and that suboptimal dietary (in particular, energy) intake of mothers does not have notable effects human milk macronutrient content [44]. Whether this is the case for maternal dietary macronutrient content or dietary patterns and HMO composition remains unknown.

Studies of dietary supplementation or specific micronutrients and their associations with HMO composition and profiles are also scarce, with two interventional studies included in this review $[18,22]$. At the very least, future trials should aim to include baseline measures and control for other sources of variance such as maternal and infant diets. When investigating pre/probiotic or symbiotic supplements, assessment of the interplay between maternal and infant gut microbiomes, as well as the milk microbiome might be valuable and relevant. Seppo et al. [22] found that probiotic supplements given during late stages of pregnancy changed the relative HMO composition, possibly by shaping the maternal gut microbiome. It is plausible that this in turn shapes the milk microbiome (via the enteromammary pathway) and successively the overall milk environment of which HMOs form a part [13]. A possible diet-related mechanism may also be that diets high in fibre-rich food groups such as fruit and wholegrains may also shape the maternal microbiome. The work by the Azad [12] and Quin [27] groups found evidence of associations between both food groups and HMO composition, and perhaps the mechanism is the interplay between the maternal diet, gut microbiome, the milk microbiome, and the HMO composition therein. Certainly, initial evidence exists for a link between maternal dietary changes, HMOs, and changes in the milk microbiome metagenomic functional capacity [42].

The evidence for associations between maternal body composition and HMO profiles may be slightly more compelling. The hypotheses behind the reported associations vary and are speculative at this stage, but center around the idea that glycosylation (and therefore HMO composition) may be influenced by maternal physiological status. The largest multi-site, international study included in this review [20] confirmed correlations between maternal body weight and/or BMI and select HMOs during lactation. Even pre-pregnancy BMI and weight showed associations with HMOs $[8,15]$, and these may be correlated with breastfeeding practices and the overall duration of breastfeeding [45]. Direct evidence linking maternal body composition and $\mathrm{HMO}$ profiles from the included studies remains limited however, and in many cases involved only singular changes in a few HMOs which 
differed across studies. It should also be noted that BMI does not seem to relate to overall breast milk quality and quantity, except in cases of severe undernutrition [46]. Further, the validity of self-reported pre-pregnancy body weight and the appropriateness of BMI as a measure of body composition during lactation is questionable. The need for standardised body composition tools beyond the BMI is clear. These tools would also need to be accurate, valid, reliable, and chosen after consideration of factors such as suitability/acceptability and cost. Measures such as dual-energy X-ray absorptiometry (DXA) and bioimpedance analysis (BIA) represent such options [47]. Despite being the current gold standard for body composition measurement, DXA was not used by any of the studies included in this review. The only study that utilised bioelectrical impedance spectroscopy (BIS) found no associations between maternal body composition and calculated HMO levels [16]. HMOs were not quantitated directly however, limiting investigations into the effect on singular or classes of HMOs. The discrepancies between, and paucity of results, certainly warrants further investigation into how maternal pre-pregnancy BMI, pregnancy weight gain, and maternal nutritional status during lactation affect HMO composition. The link between maternal dietary intake, including diet quality and dietary patterns, and body composition as they relate to HMO profiles, has also not yet been explored.

Overall, the findings from the studies included in this scoping review cautiously suggest that maternal dietary intake and body composition could affect the biosynthesis of HMOs, although this hypothesis and the mechanistic links would need to be confirmed in additional studies. One such concept to be explored is the interaction between maternal nutrition, its impact on HMO composition and the milk microbiome, and subsequently on the structure and function of the infant gut microbiome. Quin and colleagues [27] demonstrated that total dietary intake of sugars and saturated fats had a significant impact on HMO constituents and showed an additional correlation between HMO profile and infant gut microbial composition. Furthermore, a study linking maternal secretor status, HMO composition and the faecal microbiota of breastfed children show that although bacterial diversity is not affected by secretor status, the relative abundance of certain bacteria is affected [40]. A more detailed assessment of nutrient intake during lactation in particular may be required to identify (or exclude) dietary effects on $\mathrm{HMO}$ composition, preferably using quantitative methods of $\mathrm{HMO}$ analysis, valid and reliable nutrition assessment tools, and large samples of healthy mothers across different geographic settings. Little is known about the link between maternal physiological status (nutritional status in particular), and the interindividual variability of HMO levels. Further, the clinical significance of changes in HMO composition in terms of the impact on infant health (or disease) and development requires further investigation. It is hoped that modulating the $\mathrm{HMO}$ composition of human milk via modifications of the maternal diet, body composition and even the microbiome, may lead to opportunities to maximise health outcomes of breastfed infants. Potential future projects could include integrative exploration of the link between the maternal, milk, and infant microbiomes, extended therapeutic uses of HMOs, and the estimation of optimal levels of HMOs in human milk and formulas for infant health.

\section{Strengths and Limitations}

This scoping review is the first to map what is known about maternal nutritional intake and status and the profile of HMOs, and it briefly assesses the methodological and analytical approaches in the included studies. Selection bias may have been introduced because only original journal articles published in English were included. Further, the review does not include articles published after March 2020 and examines only a limited number of available scientific papers. Despite these limitations, this review is strengthened by its compliance to the PRISMA-ScR guidelines and robust search strategy conducted in multiple databases. 


\section{Conclusions}

The purpose of this review was to describe the extent of research undertaken to assess the effect of maternal dietary intake and body composition on $\mathrm{HMO}$ variability and content during established lactation. As these factors are largely modifiable, it is anticipated that the outcomes of this review will help to inform nutritional guidelines for lactating women in order to optimise the composition of bioactive oligosaccharides in human milk. Future studies should aim to follow standardised methods of milk sampling, storage, and analysis, as well as utilise robust nutritional assessment tools. Given the emerging and significant role that human milk plays in overall infant health and development, research into ways to optimise its nutritional superiority and $\mathrm{HMO}$ quality must continue to be a focus area.

\section{Update}

At the time of this review, no study had clearly demonstrated associations between the maternal diet, $\mathrm{HMO}$ composition, and the breast milk microbiome. However, a recent paper has provided some evidence in this regard, albeit with small study numbers. In a series of inpatient, single-blinded cross-over dietary intervention studies, small groups $(n=7)$ of lactating women received diets that varied according to primary carbohydrate or dietary macronutrient source. Within even a short duration of intervention, results suggest that maternal diet can modulate the profile of HMOs as well as the functional capacity, though not taxonomic composition of the milk microbiome [42]. Further evidence is sought through larger long-term dietary studies.

Author Contributions: Conceptualisation, C.B., J.M. and P.S.W.D.; methodology, J.M. and R.J.C.; formal analysis, C.B. and J.M.; writing—original draft preparation, C.B.; writing — review and editing, J.M., M.H., A.K.; supervision, P.S.W.D. and P.K.; project administration, R.J.C.; funding acquisition, C.B., M.H., A.K. and J.M. All authors have read and agreed to the published version of the manuscript.

Funding: No specific funding was obtained for this review. However, C.B. is a recipient of the Research Training Program (RTP) Stipend Scholarship, funded by the Australian Government and USC; and the Agilent Technologies Top-up/Sunshine Coast Research Scholarship (USCRS).

Conflicts of Interest: The authors declare no conflict of interest. The funders had no role in the design of the study; in the collection, analyses, or interpretation of data; in the writing of the manuscript, or in the decision to publish the results.

\section{References}

1. Ballard, O.; Morrow, A.L. Human Milk Composition. Nutrients and Bioactive Factors. Pediatric Clin. North. Am. 2013, 60, 49-74. [CrossRef] [PubMed]

2. Bode, L. Human milk oligosaccharides: Every baby needs a sugar mama. Glycobiology 2012, 22, 1147-1162. [CrossRef] [PubMed]

3. Austin, S.; Bénet, T. Quantitative determination of non-lactose milk oligosaccharides. Anal. Chim. Acta 2018, 1010, 86-96. [CrossRef] [PubMed]

4. Bzikowska-Jura, A.; Czerwonogrodzka-Senczyna, A.; Olędzka, G.; Szostak-Węgierek, D.; Weker, H.; Wesołowska, A. Maternal Nutrition and Body Composition During Breastfeeding: Association with Human Milk Composition. Nutrients 2018, 10, 1379. [CrossRef]

5. Ray, C.; Kerketta, J.A.; Rao, S.; Patel, S.; Dutt, S.; Arora, K.; Pournami, F.; Bhushan, P. Human Milk Oligosaccharides: The Journey Ahead. Int. J. Pediatr. 2019, 2019, 2390240. [CrossRef]

6. Austin, S.; De Castro, C.A.; Sprenger, N.; Binia, A.; Affolter, M.; Garcia-Rodenas, C.L.; Beauport, L.; Tolsa, J.F.; Fumeaux, C.J.F. Human milk oligosaccharides in the milk of mothers delivering term versus preterm infants. Nutrients 2019, 11, 1282. [CrossRef]

7. Wang, B.; Yu, B.; Karim, M.; Hu, H.; Sun, Y.; McGreevy, P.; Petocz, P.; Held, S.; Brand-Miller, J. Dietary sialic acid supplementation improves learning and memory in piglets. Am. J. Clin. Nutr. 2007, 85, 561-569. [CrossRef]

8. Samuel, T.M.; Binia, A.; de Castro, C.A.; Thakkar, S.K.; Billeaud, C.; Agosti, M.; Al-Jashi, I.; Costeira, M.J.; Marchini, G.; MartinezCosta, C.; et al. Impact of maternal characteristics on human milk oligosaccharide composition over the first 4 months of lactation in a cohort of healthy European mothers. Sci Rep. 2019, 9, 1-10. [CrossRef]

9. Thurl, S.; Munzert, M.; Boehm, G.; Matthews, C.; Stahl, B. Systematic review of the concentrations of oligosaccharides in human milk. Nutr. Rev. 2017, 75, 920-933. [CrossRef]

10. Sprenger, N.; Binia, A.; Austin, S. Human Milk Oligosaccharides: Factors Affecting Their Composition and Their Physiological Significance. Nestle Nutr Inst. Workshop Ser. 2019, 90, 43-56. [CrossRef] 
11. Coppa, G.V.; Gabrielli, O.; Zampini, L.; Galeazzi, T.; Ficcadenti, A.; Padella, L.; Santoro, L.; Soldi, S.; Carlucci, A.; Bertino, E.; et al. Oligosaccharides in 4 different milk groups, bifidobacteria, and ruminococcus obeum. J. Pediatr. Gastroenterol. Nutr. 2011, 53, 80-87. [CrossRef]

12. Azad, M.B.; Robertson, B.; Atakora, F.; Becker, A.B.; Subbarao, P.; Moraes, T.J.; Mandhane, P.J.; Turvey, S.E.; Lefebvre, D.L.; Sears, M.R.; et al. Human Milk Oligosaccharide Concentrations Are Associated with Multiple Fixed and Modifiable Maternal Characteristics, Environmental Factors, and Feeding Practices. J. Nutr. 2018, 148, 1733-1742. [CrossRef] [PubMed]

13. Moossavi, S.; Sepehri, S.; Robertson, B.; Bode, L.; Goruk, S.; Field, C.J.; Lix, L.M.; de Souza, R.J.; Becker, A.B.; Mandhane, P.J.; et al. Composition and Variation of the Human Milk Microbiota Are Influenced by Maternal and Early-Life Factors. Cell Host Microbe 2019, 25, 324-335.e4. [CrossRef] [PubMed]

14. Davis, J.C.C.; Lewis, Z.T.; Krishnan, S.; Bernstein, R.M.; Moore, S.E.; Prentice, A.M.; Mills, D.A.; Lebrilla, C.B.; Zivkovic, A.M. Growth and Morbidity of Gambian Infants are Influenced by Maternal Milk Oligosaccharides and Infant Gut Microbiota. Sci. Rep. 2017, 7, 40466. [CrossRef] [PubMed]

15. Ferreira, A.L.; Alves, R.; Figueiredo, A.; Alves-Santos, N.; Freitas-Costa, N.; Batalha, M.; Yonemitsu, C.; Manivong, N.; Furst, A.; Bode, L.; et al. Human milk oligosaccharide profile variation throughout postpartum in healthy women in a Brazilian cohort. Nutrients 2020, 12, 790. [CrossRef] [PubMed]

16. Gridneva, Z.; Rea, A.; Tie, W.J.; Lai, C.T.; Kugananthan, S.; Ward, L.C.; Murray, K.; Hartmann, P.E.; Geddes, D.T. Carbohydrates in human milk and body composition of term infants during the first 12 months of lactation. Nutrients 2019, 11, 1472. [CrossRef] [PubMed]

17. Jantscher-Krenn, E.; Treichler, C.; Brandl, W.; Schönbacher, L.; Köfeler, H.; Van Poppel, M.N.M. The association of human milk oligosaccharides with glucose metabolism in overweight and obese pregnant women. Am. J. Clin. Nutr. 2019, 110, 1335-1343. [CrossRef]

18. Jorgensen, J.M.; Arnold, C.; Ashorn, P.; Ashorn, U.; Chaima, D.; Cheung, Y.B.; Davis, J.C.C.; Fan, Y.M.; Goonatilleke, E.; Kortekangas, E.; et al. Lipid-based nutrient supplements during pregnancy and lactation did not affect human milk oligosaccharides and bioactive proteins in a randomized trial. J. Nutr. 2017, 147, 1867-1874. [CrossRef]

19. Larsson, M.W.; Lind, M.V.; Laursen, R.P.; Yonemitsu, C.; Larnkjær, A.; Mølgaard, C.; Michaelsen, K.F.; Bode, L. Human Milk Oligosaccharide Composition Is Associated with Excessive Weight Gain During Exclusive Breastfeeding-An Explorative Study. Front. Pediatr. 2019, 7, 297. [CrossRef]

20. McGuire, M.K.; Meehan, C.L.; McGuire, M.A.; Williams, J.E.; Foster, J.; Sellen, D.W.; Kamau-Mbuthia, E.W.; Kamundia, E.W.; Mbugua, S.; Moore, S.E.; et al. What's normal? Oligosaccharide concentrations and profiles in milk produced by healthy women vary geographically. Am. J. Clin. Nutr. 2017, 105, 1086-1100. [CrossRef] [PubMed]

21. Qiao, Y.; Feng, J.; Yang, J.; Gu, G. The relationship between dietary vitamin A intake and the levels of sialic acid in the breast milk of lactating women. J. Nutr. Sci. Vitaminol. 2013, 59, 347-351. [CrossRef] [PubMed]

22. Seppo, A.E.; Kukkonen, A.K.; Kuitunen, M.; Savilahti, E.; Yonemitsu, C.; Bode, L.; Järvinen, K.M. Association of Maternal Probiotic Supplementation with Human Milk Oligosaccharide Composition. JAMA Pediatr. 2019, 173, 286-288. [CrossRef] [PubMed]

23. Autran, C.A.; Kellman, B.P.; Kim, J.H.; Asztalos, E.; Blood, A.B.; Spence, E.C.H.; Patel, A.L.; Hou, J.; Lewis, N.E.; Bode, L. Human milk oligosaccharide composition predicts risk of necrotising enterocolitis in preterm infants. Gut 2018, 67, 1064-1070. [CrossRef]

24. Walsh, C.; Lane, J.A.; van Sinderen, D.; Hickey, R.M. Human milk oligosaccharides: Shaping the infant gut microbiota and supporting health. J. Funct. Foods 2020, 72, 104074. [CrossRef] [PubMed]

25. Wiciński, M.; Sawicka, E.; Gębalski, J.; Kubiak, K.; Malinowski, B. Human milk oligosaccharides: Health benefits, potential applications in infant formulas, and pharmacology. Nutrients 2020, 12, 266. [CrossRef] [PubMed]

26. Tricco, A.C.; Lillie, E.; Zarin, W.; O’Brien, K.K.; Colquhoun, H.; Levac, D.; Moher, D.; Peters, M.D.; Horsley, T.; Weeks, L.; et al. PRISMA Extension for Scoping Reviews (PRISMA-ScR): Checklist and Explanation. Ann. Intern. Med. 2018, 169, 467-473. [CrossRef]

27. Quin, C.; Vicaretti, S.D.; Mohtarudin, N.A.; Garner, A.M.; Vollman, D.M.; Gibson, D.L.; Zandberg, W.F. Influence of sulfonated and diet-derived human milk oligosaccharides on the infant microbiome and immune markers. J. Biol Chem 2020, 295, 4035-4048. [CrossRef] [PubMed]

28. Isganaitis, E.; Venditti, S.; Matthews, T.J.; Lerin, C.; Demerath, E.W.; Fields, D.A. Maternal obesity and the human milk metabolome: Associations with infant body composition and postnatal weight gain. Am. J. Clin. Nutr. 2019, 110, 111-120. [CrossRef]

29. Moossavi, S.; Atakora, F.; Miliku, K.; Sepehri, S.; Robertson, B.; Duan, Q.L.; Becker, A.B.; Mandhane, P.J.; Turvey, S.E.; Moraes, T.J.; et al. Integrated analysis of human milk microbiota with oligosaccharides and fatty acids in the child cohort. Front. Nutr. 2019, 6. [CrossRef]

30. Tonon, K.M.; de Morais, M.B.; Abrão, A.C.F.V.; Miranda, A.; Morais, T.B. Maternal and Infant Factors Associated with Human Milk Oligosaccharides Concentrations According to Secretor and Lewis Phenotypes. Nutrients 2019, 11, 1358. [CrossRef]

31. Long, H.A.; French, D.P.; Brooks, J.M. Optimising the value of the critical appraisal skills programme (CASP) tool for quality appraisal in qualitative evidence synthesis. Res. Methods Med. Health Sci. 2020, 1, 31-42. [CrossRef] 
32. Subbarao, P.; Anand, S.S.; Becker, A.B.; Befus, A.D.; Brauer, M.; Brook, J.R.; Denburg, J.A.; HayGlass, K.T.; Kobor, M.S.; Kollmann, T.R.; et al. The Canadian Healthy Infant Longitudinal Development (CHILD) Study: Examining developmental origins of allergy and asthma. Thorax 2015, 70, 998-1000. [CrossRef] [PubMed]

33. Thurl, S.; Munzert, M.; Henker, J.; Boehm, G.; Mller-Werner, B.; Jelinek, J.; Stahl, B. Variation of human milk oligosaccharides in relation to milk groups and lactational periods. Br. J. Nutr. 2010, 104, 1261-1271. [CrossRef] [PubMed]

34. Cabrera-Rubio, R.; Kunz, C.; Rudloff, S.; García-Mantrana, I.; Crehuá-Gaudiza, E.; Martínez-Costa, C.; Carmen Collado, M. Association of Maternal Secretor Status and Human Milk Oligosaccharides with Milk Microbiota: An Observational Pilot Study. J. Pediatr. Gastroenterol. Nutr. 2019, 68, 256-263. [CrossRef]

35. Subar, A.F.; Kirkpatrick, S.I.; Mittl, B.; Zimmerman, T.P.; Thompson, F.E.; Bingley, C.; Willis, G.; Islam, N.G.; Baranowski, T.; McNutt, S.; et al. The Automated Self-Administered 24-hour dietary recall (ASA24): A resource for researchers, clinicians, and educators from the National Cancer Institute. J. Acad Nutr Diet. 2012, 112, 1134-1137. [CrossRef]

36. Garrow, J.S.; Webster, J. Quetelet's index (W/H2) as a measure of fatness. Int J. Obes 1985, 9, 147-153.

37. Nuttall, F.Q. Body Mass Index: Obesity, BMI, and Health: A Critical Review. Nutr. Today 2015, 50, 117-128. [CrossRef]

38. Gridneva, Z.; Kugananthan, S.; Hepworth, A.R.; Tie, W.J.; Lai, C.T.; Ward, L.C.; Hartmann, P.E.; Geddes, D.T. Effect of Human Milk Appetite Hormones, Macronutrients, and Infant Characteristics on Gastric Emptying and Breastfeeding Patterns of Term Fully Breastfed Infants. Nutrients 2016, 9, 15. [CrossRef]

39. Urashima, T.; Hirabayashi, J.; Sato, S.; Kobata, A. Human milk oligosaccharides as essential tools for basic and application studies on galectins. Trends Glycosci. Glycotechnol. 2018, 30, SE51-SE65. [CrossRef]

40. Smith-Brown, P.; Morrison, M.; Krause, L.; Davies, P.S.W. Mothers secretor status affects development of childrens microbiota composition and function: A pilot study. PLOS ONE 2016, 11, 1358. [CrossRef]

41. Lee, Y.Q.; Loh, J.; Ang, R.S.E.; Chong, M.F.-F. Tracking of Maternal Diet from Pregnancy to Postpregnancy: A Systematic Review of Observational Studies. Curr. Dev. Nutr. 2020, 4, nzaa118. [CrossRef]

42. Seferovic, M.D.; Mohammad, M.; Pace, R.M.; Engevik, M.; Versalovic, J.; Bode, L.; Haymond, M.; Aagaard, K.M. Maternal diet alters human milk oligosaccharide composition with implications for the milk metagenome. Sci. Rep. 2020, 10, 1-18. [CrossRef]

43. Bravi, F.; Wiens, F.; Decarli, A.; Dal Pont, A.; Agostoni, C.; Ferraroni, M. Impact of maternal nutrition on breast-milk composition: A systematic review. Am. J. Clin. Nutr. 2016, 104, 646-662. [CrossRef] [PubMed]

44. Minato, T.; Nomura, K.; Asakura, H.; Aihara, A.; Hiraike, H.; Hino, Y.; Isojima, T.; Kodama, H. Maternal Undernutrition and Breast Milk Macronutrient Content Are Not Associated with Weight in Breastfed Infants at 1 and 3 Months after Delivery. Int J. Env. Res. Public Health 2019, 16, 3315. [CrossRef] [PubMed]

45. Castillo, H.; Santos, I.S.; Matijasevich, A. Maternal pre-pregnancy BMI, gestational weight gain and breastfeeding. Eur. J. Clin. Nutr. 2016, 70, 431-436. [CrossRef] [PubMed]

46. Prentice, A.M.; Goldberg, G.R.; Prentice, A. Body mass index and lactation performance. Eur J. Clin. Nutr 1994, 48 (Suppl. S3), S78-S86; discussion S86-S89.

47. Marra, M.; Sammarco, R.; De Lorenzo, A.; Iellamo, F.; Siervo, M.; Pietrobelli, A.; Donini, L.M.; Santarpia, L.; Cataldi, M.; Pasanisi, F.; et al. Assessment of Body Composition in Health and Disease Using Bioelectrical Impedance Analysis (BIA) and Dual Energy X-Ray Absorptiometry (DXA): A Critical Overview. Contrast Media Mol. Imaging 2019, 2019, 1-9. [CrossRef] 